\title{
Notch promotes tumor metastasis in a prostate-specific Pten-null mouse model
}

\author{
Oh-Joon Kwon, ${ }^{1}$ Li Zhang, ${ }^{1}$ Jianghua Wang, ${ }^{2}$ Qingtai Su, ${ }^{1}$ Qin Feng, ${ }^{1}$ Xiang H.F. Zhang, ${ }^{1,3,4}$ Sendurai A. Mani, ${ }^{5}$ Robia Paulter, ${ }^{6}$ \\ Chad J. Creighton, ${ }^{4,7}$ Michael M. Ittmann, ${ }^{2,4,8}$ and Li Xin ${ }^{1,2,4}$ \\ 'Department of Molecular and Cellular Biology, ${ }^{2}$ Department of Pathology and Immunology, ${ }^{3}$ Lester and Sue Smith Breast Center, and ${ }^{4}$ Dan L. Duncan Cancer Center, Baylor College of Medicine, \\ Houston, Texas, USA. ${ }^{5}$ Department of Translational Molecular Pathology, Division of Pathology and Laboratory Medicine, The University of Texas MD Anderson Cancer Center, Houston, Texas, USA. \\ ${ }^{6}$ Department of Molecular Physiology, ${ }^{7}$ Department of Medicine, and ${ }^{8}$ Michael E. DeBakey Department of Veterans Affairs Medical Center, Baylor College of Medicine, Houston, Texas, USA.
}

\begin{abstract}
Although Notch signaling is deregulated in prostate cancer, the role of this pathway in disease development and progression is not fully understood. Here, we analyzed 2 human prostate cancer data sets and found that higher Notch signaling correlates with increased metastatic potential and worse disease survival rates. We used the Pten-null mouse prostate cancer model to investigate the function of Notch signaling in the initiation and progression of prostate cancer. Disruption of the transcription factor RBPJ in Pten-null mice revealed that endogenous canonical Notch signaling is not required for disease initiation and progression. However, augmentation of Notch activity in this model promoted both proliferation and apoptosis of prostate epithelial cells, which collectively reduced the primary tumor burden. The increase in cellular apoptosis was linked to DNA damage-induced p53 activation. Despite a reduced primary tumor burden, Notch activation in Pten-null mice promoted epithelial-mesenchymal transition and FOXC2-dependent tumor metastases but did not confer resistance to androgen deprivation. Notch activation also resulted in transformation of seminal vesicle epithelial cells in Pten-null mice. Our study highlights a multifaceted role for Notch signaling in distinct aspects of prostate cancer biology and supports Notch as a potential therapeutic target for metastatic prostate cancer.
\end{abstract}

\section{Introduction}

Notch is an evolutionarily conserved signaling pathway that is crucial for tissue development and homeostasis $(1,2)$. Deregulation of Notch signaling has been shown to play critical roles in disease initiation and progression in many cancer models $(3,4)$. The pro-oncogenic role of Notch in malignancies such as $\mathrm{T}$ cell acute lymphoblastic leukemia has been clearly defined (5). This is not only implied by frequent occurrences of genetic alterations that lead to elevated Notch activity, but also directly demonstrated by genetic studies using various mouse models. In contrast, genetic alterations in Notch signaling components are rare in prostate cancer on the basis of current genomic analysis (6). Nevertheless, a consensus has been reached that Notch signaling is deregulated during prostate carcinogenesis. For examples, several tissue microarray analyses have shown that Notch receptors as well as the Notch ligand JAG1 are upregulated in advanced prostate cancer specimens (7-9). However, the role of Notch signaling in prostate cancer remains inadequately defined and is often controversial (10).

Some studies implicated Notch as a pro-oncogenic signaling pathway in prostate cancer. Notch activity is positively correlated with the osteomimetic property of metastatic prostate cancer cells (11), and is enriched in prostate cancer stem cells and chemoresistant prostate cancer cells $(12,13)$. Downregulation of Notch ligands and receptors in prostate cancer cell lines suppresses pros-

Conflict of interest: The authors have declared that no conflict of interest exists. Submitted: December 9, 2015; Accepted: April 22, 2016.

Reference information: J Clin Invest. 2016;126(7):2626-2641. doi:10.1172/JCI84637. tate cancer cell proliferation in vitro (14-16). Finally, we showed that prostate-specific activation of Notch signaling promotes proliferation of adult murine prostate luminal epithelial cells and confers the capacity for anoikis resistance (17). In contrast, other studies suggest that Notch is tumor suppressive. Overexpression of the Notch intracellular domain (NICD) suppresses prostate cancer proliferation in vitro (18). Notch activation inhibits neuroendocrine differentiation of human prostate cancer cells in vitro (19). Finally, Notch downstream targets HEY1/2 and HEYL have been shown to act as androgen receptor corepressors $(20,21)$.

Most of the above conclusions were obtained through in vitro studies using established prostate cancer cell lines, or by correlative immunohistological analyses of Notch signaling components in human prostate cancer specimens. These controversies may be caused by many factors, such as the variability of prostate cancer cell lines and the lack of specificities of the siRNAs and antibodies used in those studies. Therefore, genetic studies using mouse models provide an alternative approach to determine the role of Notch in prostate cancer in a defined genetic context. Herein, we manipulated Notch activity using genetic approaches in a Pten-null mouse model for prostate cancer and examined how initiation and progression of prostate cancer were affected. Our results reveal that Notch signaling is not required for the initiation of Pten-null-induced prostate cancer. However, Notch activation promotes tumor cell proliferation and metastasis, and also induces cell apoptosis in the Ptennull mouse model for prostate cancer. This study highlights a multifaceted impact of Notch signaling on different aspects of prostate cancer cell biology. 
A
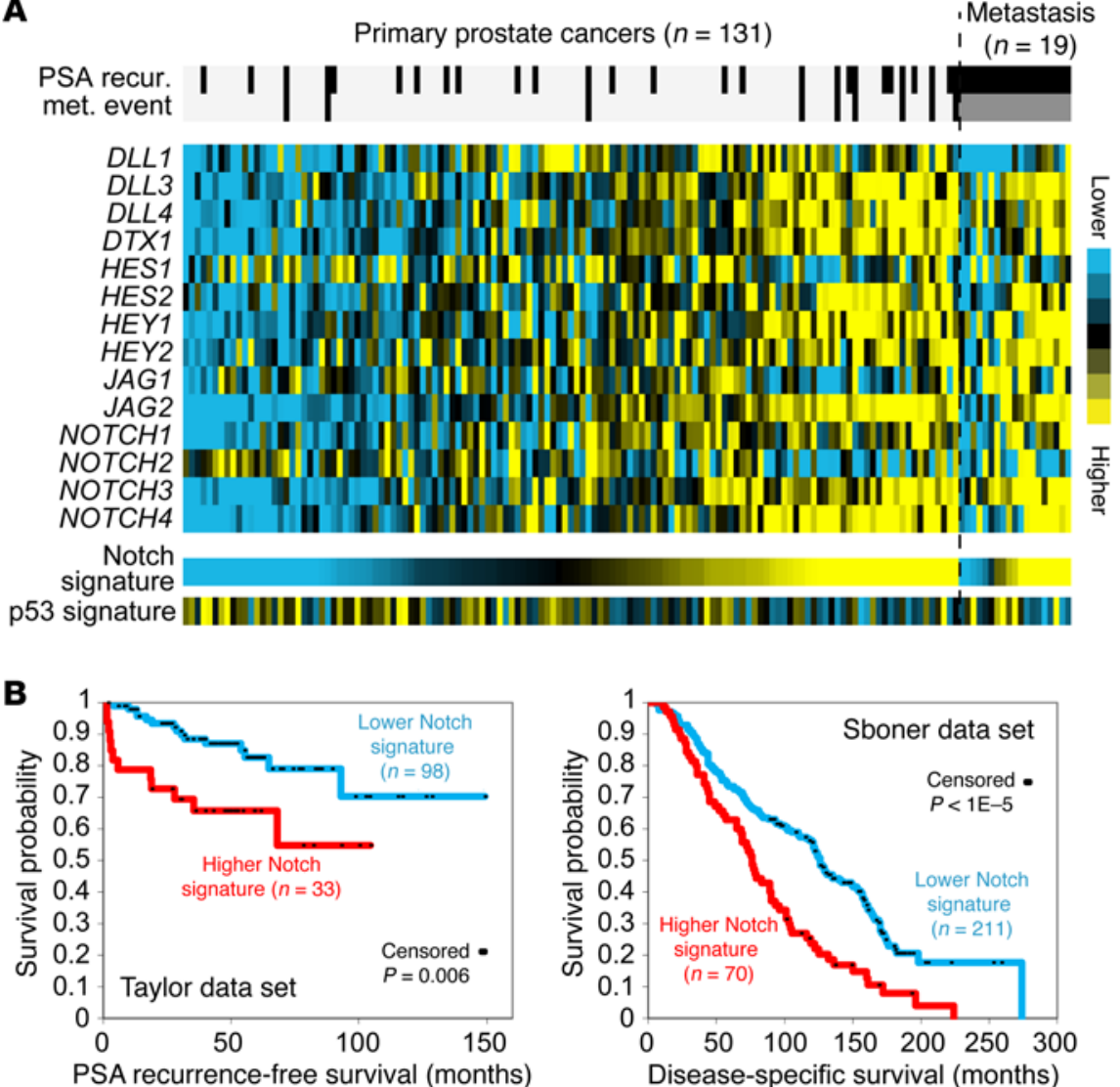

C

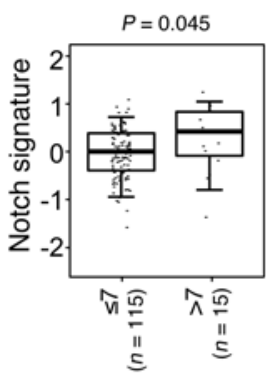

Gleason grade

(Taylor)

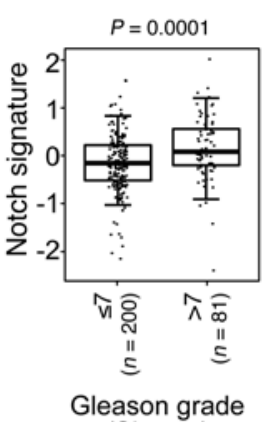

(Sboner)
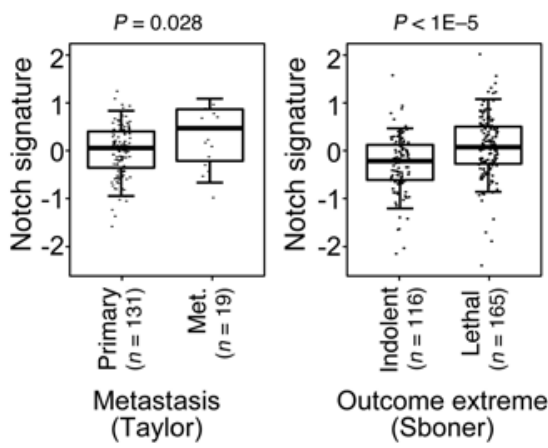

Outcome extreme (Sboner)

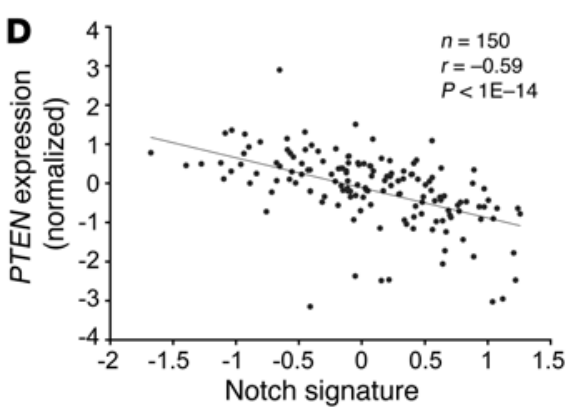

Results

Higher Notch signaling predicts poor clinical outcomes in human prostate cancer patients. To determine the correlation between Notch signaling and the progression of human prostate cancer, we defined a Notch signature score by taking the average of the nor-
Figure 1. Higher Notch signaling predicts poor clinical outcomes. (A) Expression heat map of mRNA expression for major Notch signaling components in the Taylor data set of prostate cancer specimens (22). Notch signature score was generated by averaging of the normalized expression values of the genes shown. A gene signature of p53 transcriptional targets (http://p53.iarc.fr/ TargetCenes.aspx) is inversely correlated with the Notch signature as shown. (B) Kaplan-Meier plots for correlations of Notch signature with prostate cancer recurrence in the Taylor data set (22) (left) and survival in the Sboner data set (23) (right). Log-rank test: $P<1 \times 10^{-5}$. (C) Box plots show correlations of Notch signature with increasing Gleason grade, metastatic potential, and lethal outcome. $P$ values are by $t$ test. Box plots represent $5 \%, 25 \%, 75 \%$, median, and $95 \%$. (D) Scatter plot shows an inverse correlation between Notch signature and PTEN expression levels (Spearman's correlation, $\left.r=-0.59, P<1 \times 10^{-10}\right)$.

malized mRNA expression values of major Notch signaling components in expression profiling data sets of human prostate cancer specimens $(22,23)$. The major Notch signaling components include the Notch ligands (JAG1, JAG2, DLL1, DLL3, DLL4), receptors (NOTCH1, 2, 3, 4), and several typical downstream targets (HES1, HES2, HEY1, HEY2, DTX1). Figure 1, A and B, shows that patients with a relatively higher Notch signature score (the top quarter) in primary prostate cancer specimens are more likely to develop recurrent disease in the data set of Taylor et al. (22) (log-rank test, $P<0.005)$. Interestingly, the Notch signature score is inversely correlated with expression of $\mathrm{p} 53$ transcriptional targets (Figure 1A; Pearson's $r=-0.263, P=0.001$ ). A higher Notch signature also predicts an increased probability of succumbing to the disease in the Swedish prostate cancer data set by Sboner et al. (23) (Figure 1B). In addition, as shown in Figure 1C, Notch signature is significantly correlated with increasing Gleason grade in both data sets, significantly correlated with the metastatic potential in the Taylor data set, and significantly higher in the lethal group $(n=116)$ versus the indolent group $(n=165)$ in the Sboner data set. In summary, these analyses support that augmented Notch signaling is associated with advanced prostate cancer.

Loss of function of the tumor suppressor PTEN is closely associated with prostate cancer progression (24). The Notch signature score is inversely correlated with the expression level of PTEN (Figure 1D; Pearson correlation, Spearman's $\left.r=-0.53, P<1 \times 10^{-14}\right)$. Though Notch signaling has been shown to suppress Pten expression (25), genetic and epigenetic changes 
A

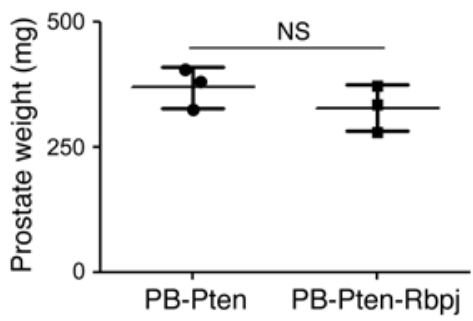

C

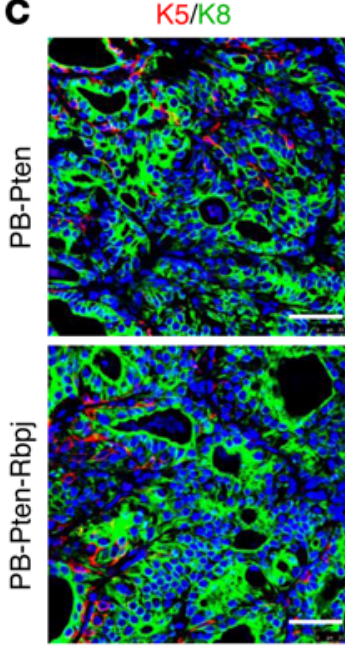

D

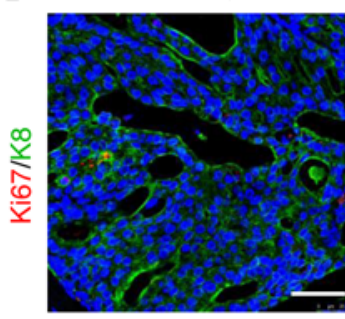

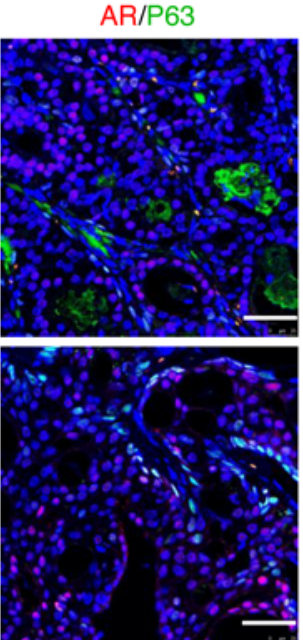

PB-Pten-Rbpj

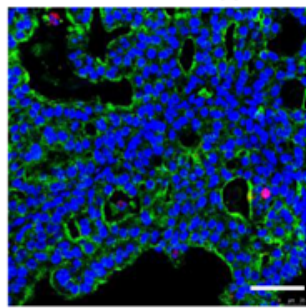

B

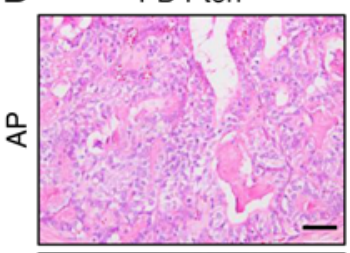

PB-Pten
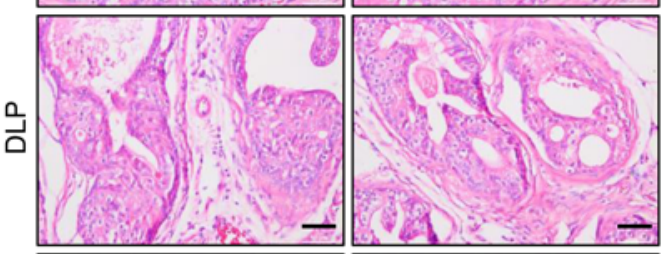

皮

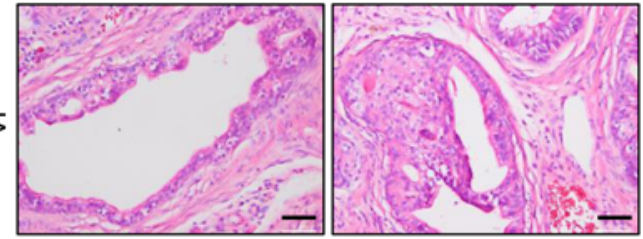

E

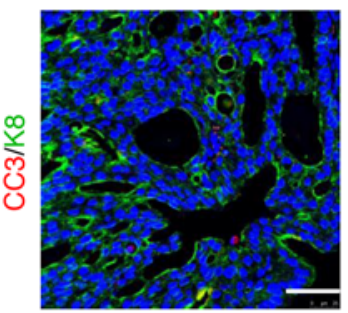

PB-Pten-Rbpj
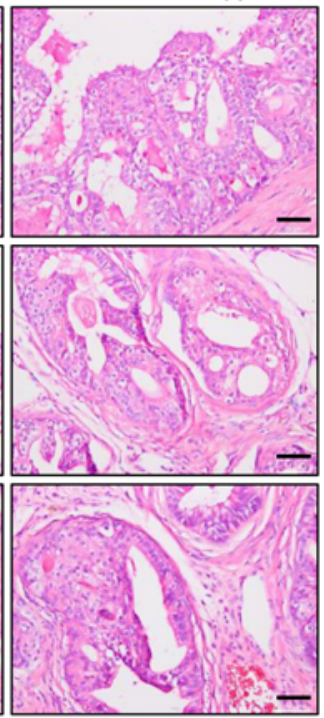

Figure 2. Notch signaling is dispensable for initiation and progression of prostate cancer in the Pten-null model. (A) Bar graph shows means \pm SD of prostate weight of 1-year-old PB-Pten and PB-Pten-Rbpj mice. $n=3$ per group. No significant difference is noted between the 2 groups (Student's $t$ test). (B) H\&E staining of anterior (AP), dorsolateral (DLP), and ventral (VP) prostate tissues from 1-year-old PB-Pten and PB-Pten-Rbpj mice. (C-E) Coimmunostaining of K5 and K8, P63, and AR (C); Ki-67 and K8 (D); and cleaved caspase 3 (CC3) and K8 (E) of prostate tissues from 1-year-old PB-Pten and PB-PtenRbpj mice. Scale bars: $50 \mu \mathrm{m}$.

are the major causes for the decreased Pten expression in prostate cancer (22). Therefore, we reasoned that the inverse correlation implies a cooperative or synergistic interaction between the 2 signaling pathways, and sought to use a prostate-specific Pten-null mouse model (26) to investigate how Notch signaling affects the progression of prostate cancer and initiates metastasis.

Notch signaling is dispensable for initiation and progression of the Pten-null mouse model for prostate cancer. RBPJ is the transcription factor essential for the activation of canonical Notch signaling (27). We showed previously that disrupting $R b p j$ in adult murine prostate epithelial cells does not impair organ morphogenesis and homeostasis (28). To determine whether Notch signaling is essential for the initiation and progression of prostate cancer in the Pten-null model, we generated a cohort of ARR2PB-Cre Pten ${ }^{f / f}$; $R b p j^{i l / f l}$ and ARR2PB-Cre Pten ${ }^{f l / l}$ mice (hereafter referred to as the PB-Pten-Rbpj and PB-Pten mice, respectively). At 1 year of age, there was no significant difference in prostate weights between the PB-Pten-Rbpj and the control PB-Pten mice (Figure 2A). Mice in both groups developed high-grade prostatic intraepithelial neoplasia (PIN) lesions and prostate adenocarcinoma with no distal metastasis (Figure 2B). IHC staining of various lineage markers also indicated no difference in lineage composition between the tumors in the 2 groups (Figure 2C). Finally, the proliferative and apoptotic indices were also comparable between the 2 groups (Figure 2, D and E, and Supplemental Figure 1; supplemental material available online with this article; doi:10.1172/JCI84637DS1). Collectively, these results show that canonical Notch signaling is dispensable for the initiation and progression of prostate cancer in the Pten-null mouse model for prostate cancer.

Notch activation shortens the lifespan of prostate-specific Ptendeletion mice. We then sought to investigate how Notch activation affects disease progression in the PB-Pten model. A ROSA26- 
A

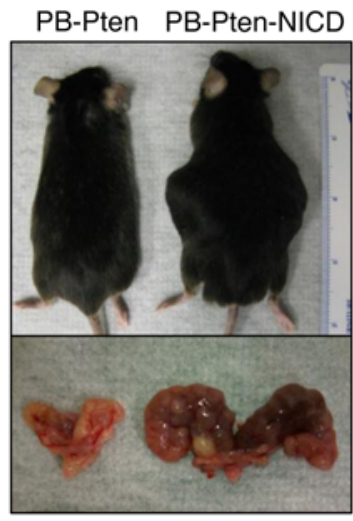

C

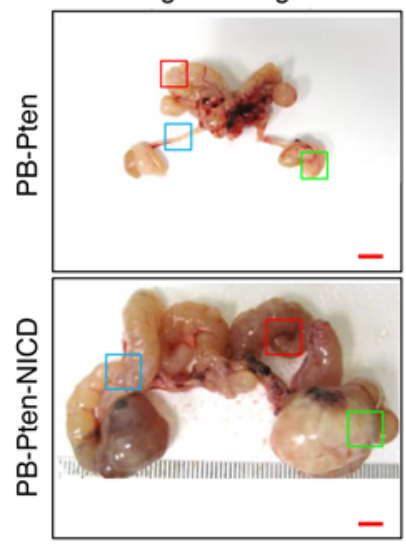

B

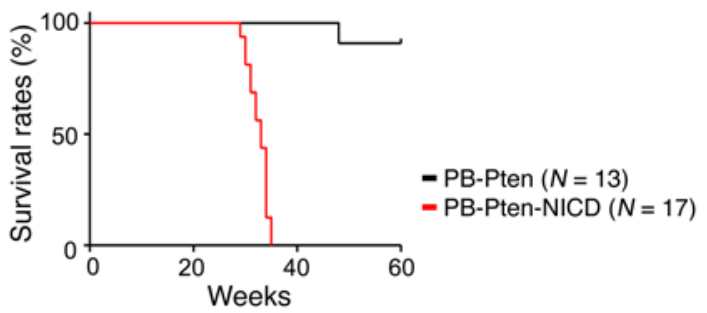

Figure 3. Notch activation shortens lifespan of PB-Pten mice. (A) Images of PB-Pten and PB-Pten-NICD mice (top) and dissected urogenital organs (bottom). (B) Kaplan-Meier survival curve of PB-Pten and PB-Pten-NICD mice. Log-rank test: $P<1 \times 10^{-4}$. (C) Transillumination images of urogenital systems and H\&E staining of epididymides, vasa deferentia, and seminal vesicles of PB-Pten and PB-Pten-NICD mice. Red bars: 5 mm; black bars: $100 \mu$ m.

LSL-NICD transgenic line enables the expression of the active Notch1 intracellular domain at the endogenous ROSA26 locus upon excision of a floxed transcriptional stop signal (29). We showed previously that prostate-specific expression of NICD (the ARR2PB-Cre ROSA26-LSL-NICD ${ }^{\mathrm{Mut} / \mathrm{Wt}}$ model) led to the formation of PIN II lesions but is insufficient to transform prostate cells or drive distal metastasis even at the age of 1.5 years (17). We bred ROSA26-LSL-NICD, ARR2PB-Cre (30), and Pten conditional mice (26) and generated a cohort of ARR2PB-Cre Pten ${ }^{f / f}$ ROSA26-LSL$\mathrm{NICD}^{\mathrm{Mut} / \mathrm{Wt}}$ and ARR2PB-Cre Pten ${ }^{A / f l}$ mice (hereafter referred to as the PB-Pten-NICD and PB-Pten mice, respectively). As shown in Figure 3A, all the PB-Pten-NICD mice had increased abdominal girth by $30-40$ weeks of age, and displayed reduced mobility and decreased alertness. These mice had to be euthanized based on the policies of the IACUC at Baylor College of Medicine (Figure 3B). In contrast, most of the PB-Pten littermates were still healthy by 60 weeks when the experiment was terminated. Dissection revealed that the increased abdominal girth of the PB-Pten-NICD mice is mainly due to enlarged seminal vesicles, vasa deferentia, and epididymides. The vas deferens and epididymis often consisted of cystic structures with empty lumens surrounded by epithelia with focal hyperplasia. In comparison, seminal vesicles, especially their tips, were often fully packed with neoplastic cells (Figure 3C). These phenotypes agree with the report that the probasin promoter is also active in these organs (30). However, these phenotypes were rarely noted in the PB-Pten mice at this age or in the ARR2PB-ROSA26-LSL-NICD mice even at the age of 1.5 years (Supplemental Figure 2), suggesting that the phenotypes are the results of interaction between the two signaling.

Notch activation results in a reduction of primary prostate tumor burden. Surprisingly, the prostate tissues of PB-Pten-NICD mice were much smaller and sometimes difficult to identify by the time the experiment was terminated. Figure $4 \mathrm{~A}$ shows that the average prostatic volume of the PB-Pten-NICD mice was already $30 \%$ smaller than that of the age-matched PB-Pten mice at 16 weeks of age by MRI analysis, which was further corroborated by prostate weights (Figure 4B). A gene expression microarray analysis was performed using prostate tissues of 16-week-old PB-Pten-NICD and PB-Pten mice. Out of approximately 60,000 probes, we identified 709 probes with 1.4- or greater fold change in expression. As expected, Notch1 and its downstream target genes (Hey1, Jag1, Nrarp, Ccnd1, Ccnd2) were all upregulated in the PB-Pten-NICD group, which is further corroborated by quantitative RT-PCR (qRT-PCR) analysis (Figure 4C). Western blot analysis shows that the expression level of NICD in the prostate tissues of PB-PtenNICD mice is approximately 3 -fold that in the human prostate cancer cell line PC3 (Figure 4D), indicating that the increased level of Notch activation in this model is disease relevant.

H\&E staining reveals that PB-Pten-NICD mice at 10-16 weeks of age developed papillary projections that form cribriform pat- 
A
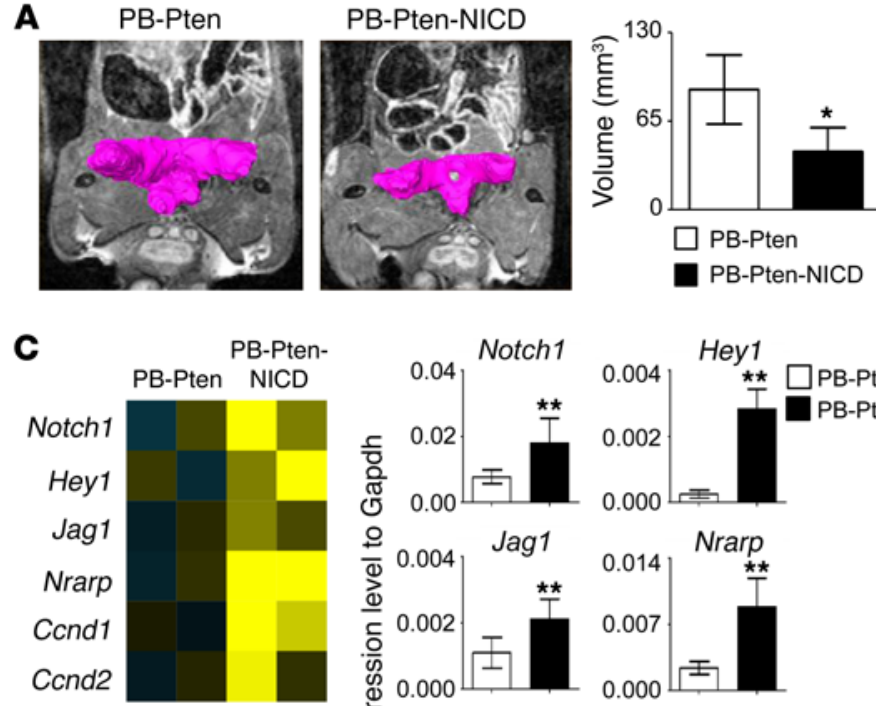

Expr. fold change $1 / 2$
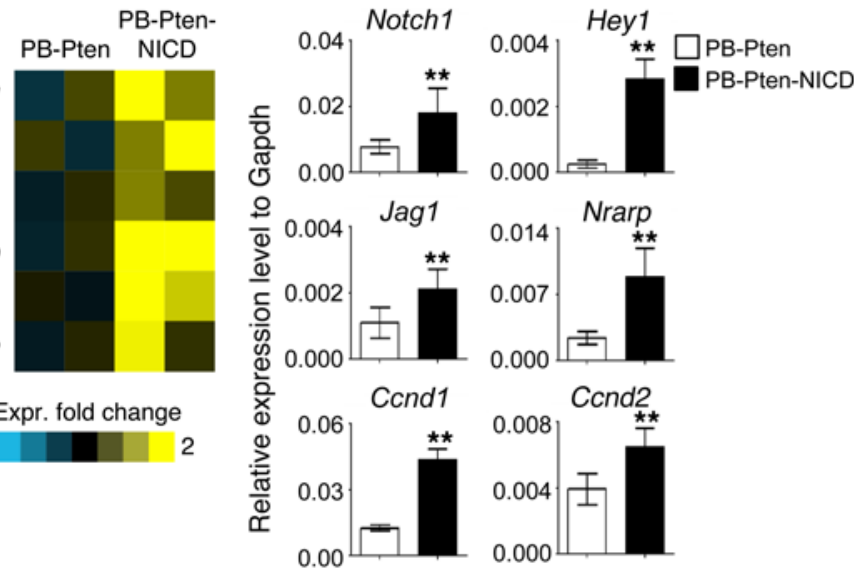

E

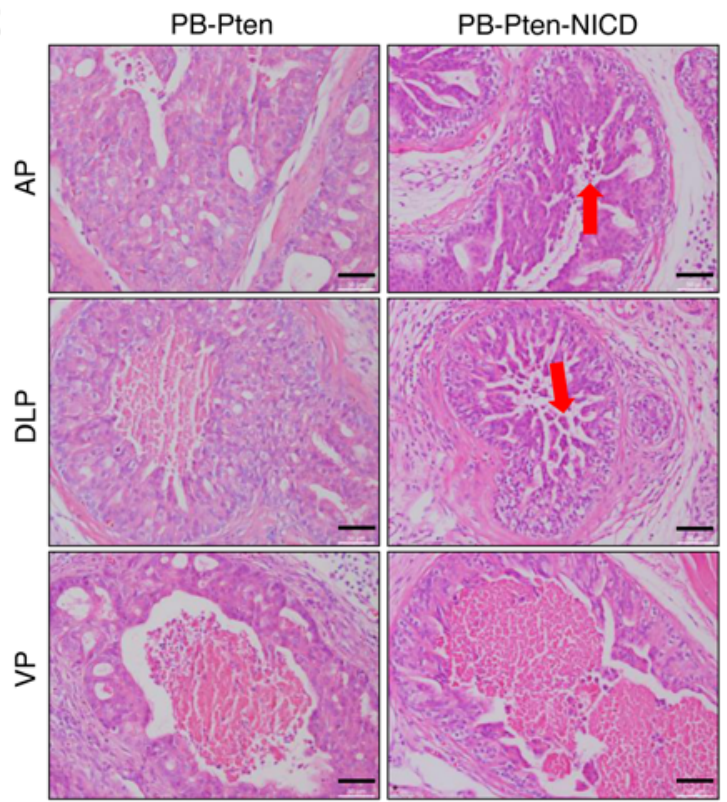

$\mathbf{F}$

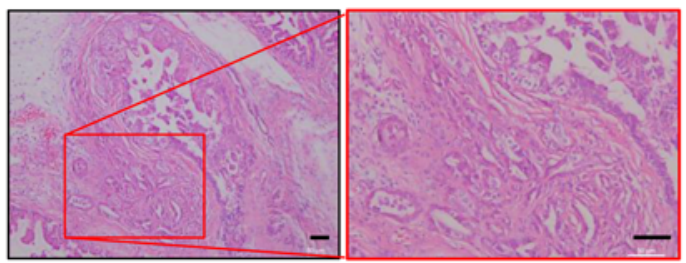

B
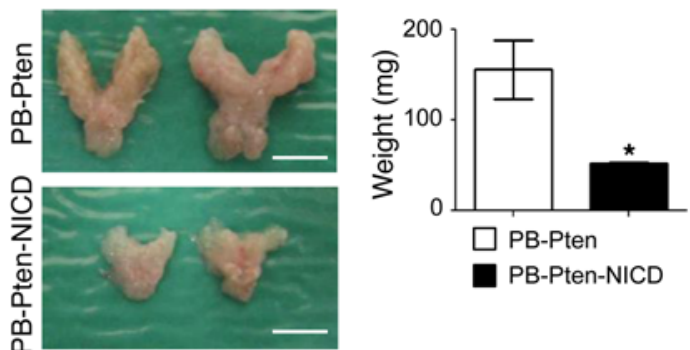

D

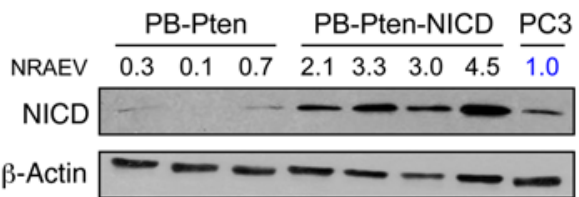

G

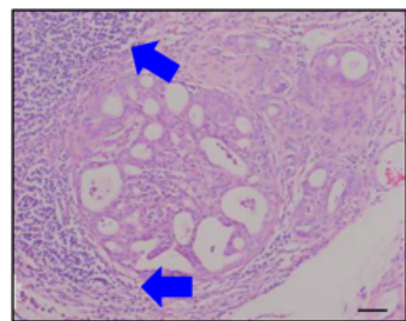

H PB-Pten

PB-Pten-NICD
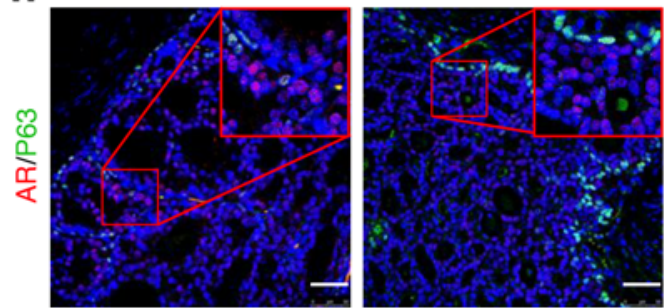

I

PB-Pten

PB-Pten-NICD
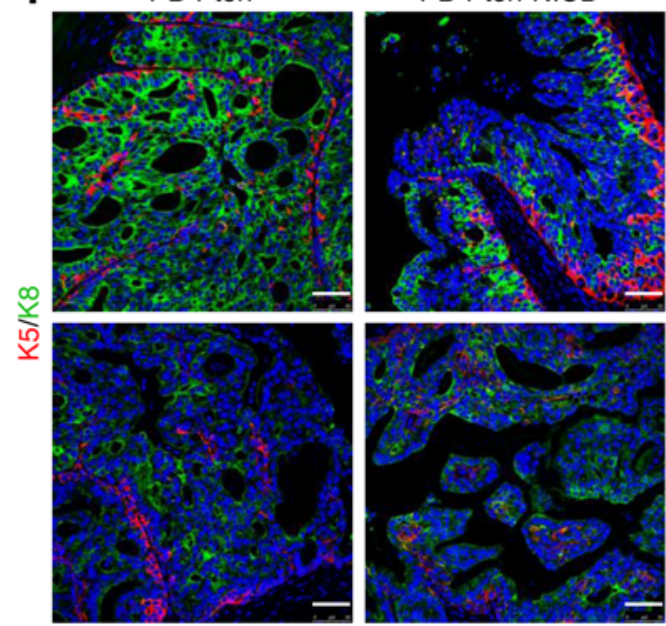

Figure 4. Notch activation results in a reduction of primary tumor burden in the Pten-null model. (A) Magnetic resonance imaging of prostate tissues in PB-Pten and PB-Pten-NICD mice at 16 weeks of age. Bar graphs show means \pm SD of tumor volume from 3 mice. ${ }^{*} P<0.05$ by Student's $t$ test. (B) Representative images of prostate tissues from PB-Pten and PB-Pten-NICD mice at 16 weeks of age. Bar graphs show means \pm SD of prostate weight from 3 mice per group. ${ }^{*} P<0.05$ by Student's $t$ test. Scale bar: $5 \mathrm{~mm}$. (C) Heat map from microarray data shows upregulation of representative Notch target genes in PB-Pten-NICD mouse prostates. qRT-PCR validates expression changes of representative genes in microarray analysis from 3 different samples per group. ${ }^{* *} P<0.001$ by Student's $t$ test. (D) Western blot analysis of NICD in prostate tissues of 16-week-old mice. Each lane represents an independent specimen. NRAEV, normalized relative arbitrary expression value by $\beta$-actin. (E) H\&E staining of anterior (AP), dorsolateral (DLP), and ventral (VP) prostate tissues from 16-week-old PB-Pten and PB-Pten-NICD mice. Red arrows point to acini inside lumen. (F) H\&E staining shows prostate adenocarcinoma in 32-week-old PB-Pten-NICD mice. (C) H\&E staining shows lymphocytic infiltration (blue arrows) in prostate tumor tissues in 32-week-old PB-Pten-NICD mice. (H and I) IHC analyses of P63 and AR (H), K5 and K8 (I) in prostate tissues of 16-week-old PB-Pten and PB-Pten-NICD mice. Scale bars: $50 \mu \mathrm{m}$. 
A

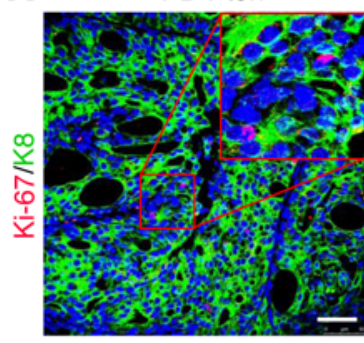

PB-Pten

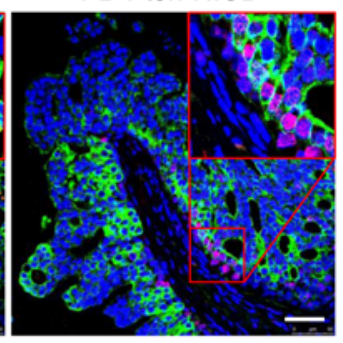

B

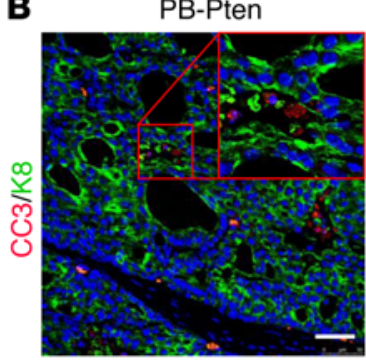

PB-Pten-NICD

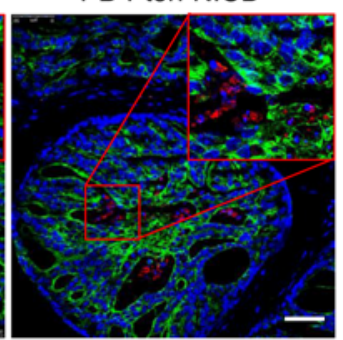

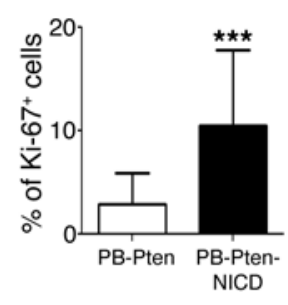

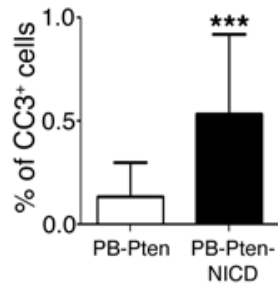

C

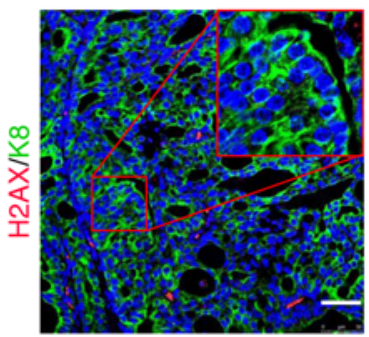

PB-Pten
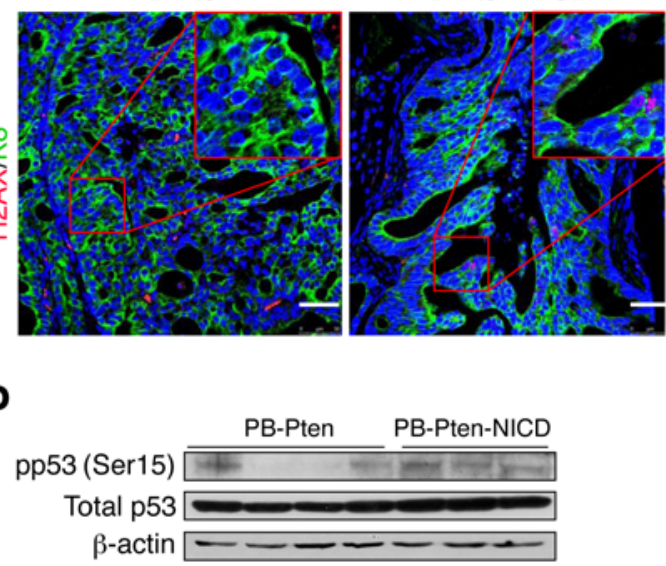
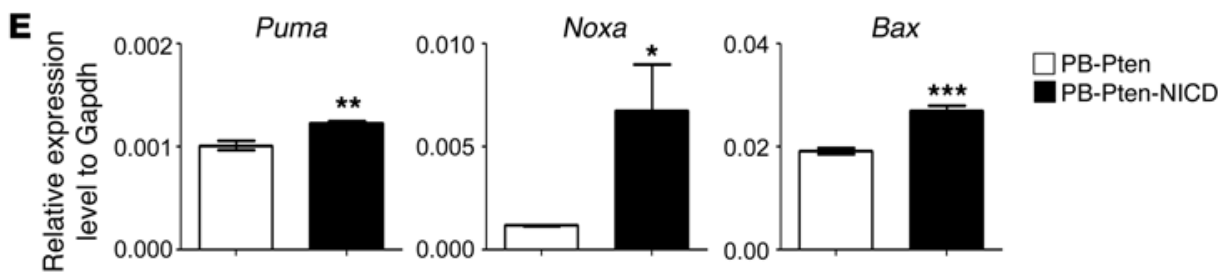

Figure 5. Notch activation promotes both proliferation and apoptosis of Pten-null prostate cancer cells. (A and B) Coimmunostaining of Ki-67 and K8 (A) and cleaved caspase 3 (СС3) and K8 (B) in 16-week-old PB-Pten and PB-Pten-NICD mouse prostates. Bar graphs show means \pm SD of Ki-67+ and CC ${ }^{+}$cells in 3 mice per group. ${ }^{* *} P<0.001$ by Student's $t$ test. (C) Coimmunostaining of pH2AX and K8 in 16-week-old PB-Pten and PB-Pten-NICD mouse prostates. (D) Western blot analysis of pSer15 p53 and total p53 in prostate tissue lysates from 16-week-old PB-Pten and PB-Pten-NICD mice. (E) qRT-PCR analysis of 3 p53 target genes in prostate tissues of 16 -week-old PB-Pten and PB-Pten-NICD mice. $n=3$ per group. ${ }^{*} P<0.05,{ }^{* *} P<0.01,{ }^{* *} P<0.001$ by Student's $t$ test. Scale bars: $50 \mu \mathrm{m}$.

terns in all prostatic lobes (Figure 4E). In addition, there are islands of acinar-like structures inside the luminal space (red arrows, Figure $4 \mathrm{E}$ ), which likely reflect the cross-section views of papillary projections. In contrast, in PB-Pten mice at 16 weeks of age, the anterior prostatic lobes are completely filled with hyperplastic cells, while the ventral and dorsolateral prostatic lobes contain empty lumen surrounded by a thick layer of epithelial cells packed tightly without prominent papillary structures protruding towards the lumen (Figure 4E). All PB-Pten-NICD mice developed adenocarcinoma by the age of 30-40 weeks (boxed area, Figure 4F), and cystic dilation of the glandular lumens was frequently noted. The other distinctive phenotype observed in PB-Pten-NICD mice at 30-40 weeks of age is prominent lymphocytic inflammation at full penetrance (blue arrows, Figure $4 G$ ).

Immunostaining analysis shows that Pten was deleted efficiently in prostate epithelial cells in both PB-Pten and PB-PtenNICD mice, suggesting that NICD does not interfere with the activity of the probasin promoter (Supplemental Figure 3). Although Notch activation in prostate epithelial cells is capable of activating AKT (17), the activation is weaker compared with that resulting from Pten deletion (Supplemental Figure 4), which further supports a potential synergy between the two signaling. The androgen receptor is expressed in the prostate tissues of both PB-Pten-NICD and PB-Pten mice (Figure $4 \mathrm{H}$ and Supplemental Figure 5A). More P63-expressing basal cells as well as K5-expressing cells are present in the prostate tissues of PB-Pten-NICD mice (Figure 4, $\mathrm{H}$ and I, and Supplemental Figure 5B). In the acinar islands inside the luminal spaces, $\mathrm{K}^{+}$cells are localized in the center (bottom right panel, Figure 4I), further supporting that these structures reflect the cross-section views of the papillary projections.

Notch activation promotes both proliferation and apoptosis of prostate cancer cells. Ki-67 staining shows that the proliferative index in the prostate tissues of the PB-Pten-NICD mice is 4.9fold greater than that of the PB-Pten mice (Figure 5A). This is also consistent with our previous report showing that Notch activation is capable of promoting epithelial cell proliferation (17). On the other hand, IHC analysis of the cleaved caspase 3 shows that the apoptotic index in the prostate tissues of PB-Pten-NICD mice was also increased 3.9-fold (Figure 5B). Figure 5C and Supplemental Figure 5C show a dramatic increase in phosphorylated Ser139 of the histone variant $\mathrm{H} 2 \mathrm{AX}(\gamma \mathrm{H} 2 \mathrm{AX})$ in the prostate tissues of PBPten-NICD mice, suggestive of excessive DNA damage. p53 activity was elevated in the PB-Pten-NICD prostate tissues as indicated by increased Ser15 phosphorylation (Figure 5D) and the increased 
A PB-Pten $(N=13)$ PB-Pten-NICD $(N=17)$
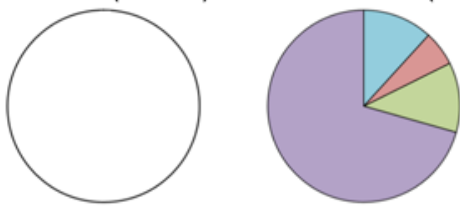

$\square$ No met

$\square$ Lung-met only

$\square$ Liver-met only

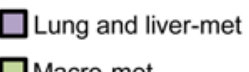

$\square$ Macro-met

B
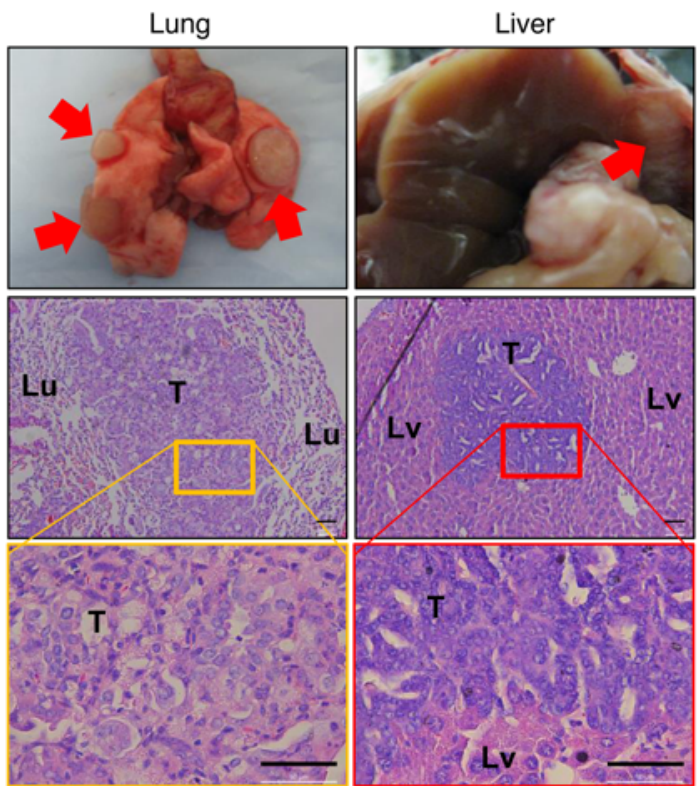

C

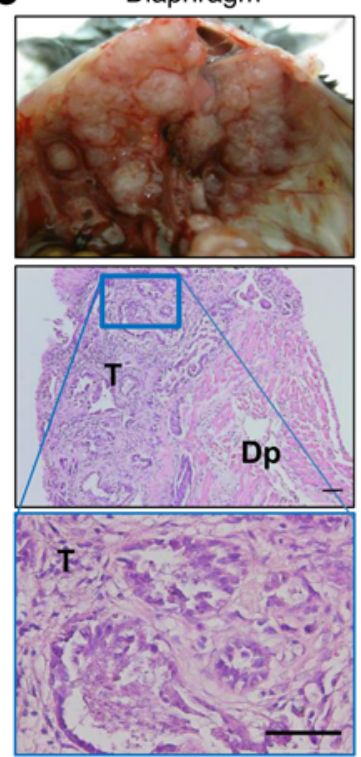

Figure 6. Notch activation promotes metastatic diseases in the Pten-null model. (A) Pie charts summarize incidence of distal metastases in PB-Pten and PB-Pten-NICD mice. (B) Transillumination images of lung (Lu) and liver (Lv) with focal metastatic nodules (top) and H\&E staining of the metastatic lesions (lower panels). (C) Transillumination image (top) and H\&E staining (lower panels) of a diaphragm (Dp) colonized with metastatic lesions. T, tumor. (D) IHC analysis of pAKT, GFP, and AR in lung and liver metastases. (E) $\mathrm{IHC}$ analysis of $\mathrm{K} 5$ and $\mathrm{K} 8$ in lung and liver metastases. Scale bars: $50 \mu \mathrm{m}$.
D
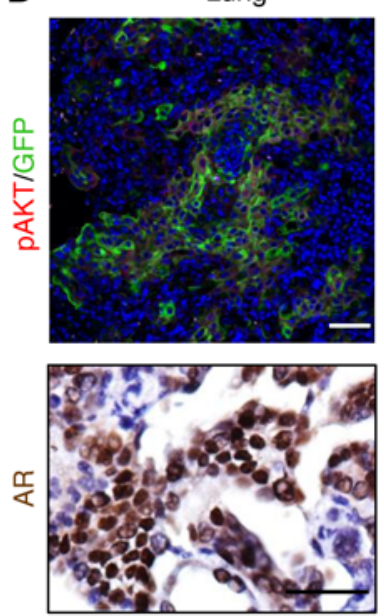

Liver
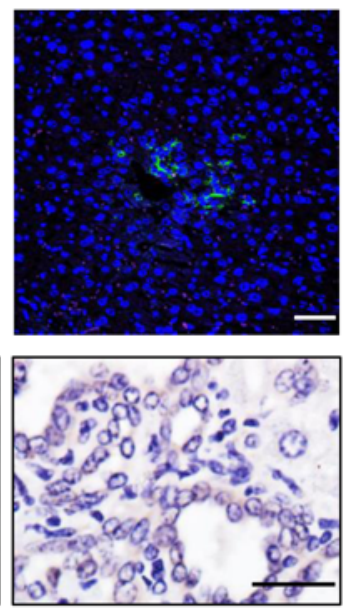

E
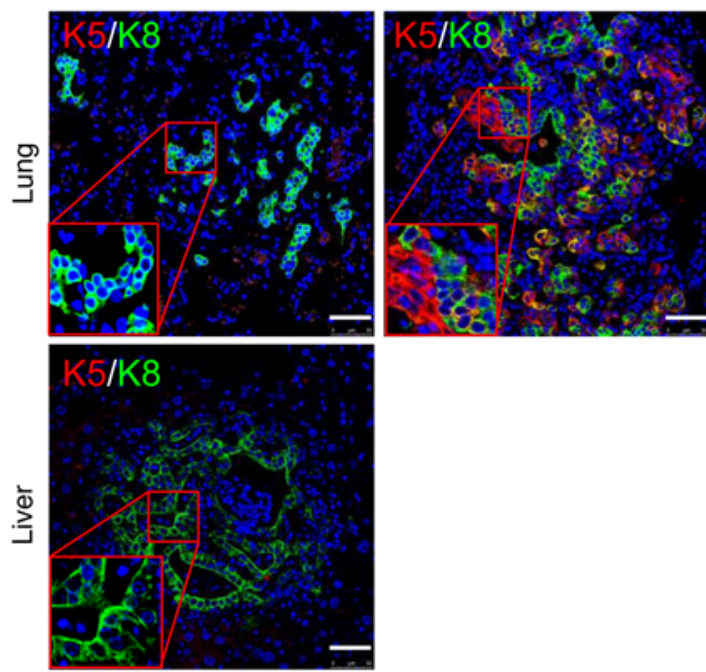

expression of p53 downstream targets Puma, Noxa, and Bax (Figure 5E). These results suggest that DNA damage-induced p53 activation contributes to the increased apoptosis. Loss of function of p53 should alleviate apoptosis and confer growth advantage, which explains why Notch signaling is usually augmented in human prostate cancer specimens with a lower p53 activity (Figure 1A). In summary, Notch activation in Pten-null prostate cancer cells promotes both proliferation and apoptosis, which collectively lead to a reduction in primary tumor burden.

Notch activation induces tumor metastasis in the Pten-null model. Despite smaller primary prostate tumor burdens, distal metastases in major internal organs including lung and liver were observed in all PB-Pten-NICD mice collected at the age of 30-40 weeks ( $n=17$ ) (Figure 6, A and B). Notably, in 2 of the mice, tumor cells appeared to metastasize by directly disseminating into the peritoneal cavity, resulting in extensive macrometastases associated with all major peritoneal organs and even to the diaphragms (Figure 6C). In contrast, none of the PB-Pten mice $(n=13)$ that we analyzed at the age of 60 weeks developed distal metastasis in major internal organs (Figure 6A).

To facilitate detection of metastatic prostate cancer cells, an mTmG fluorescence reporter allele (31) was introduced (hereaf- 
A
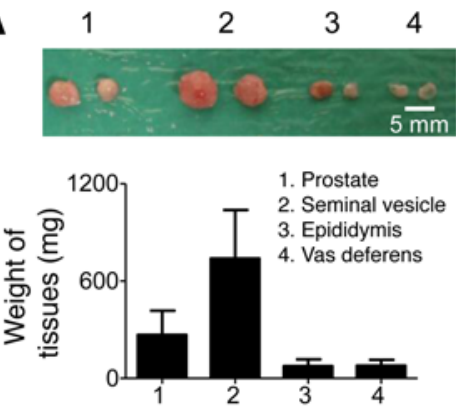

B

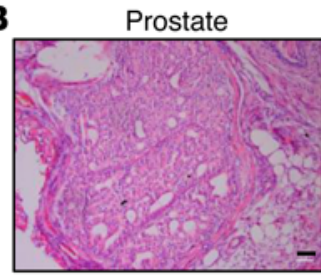

Epididymis

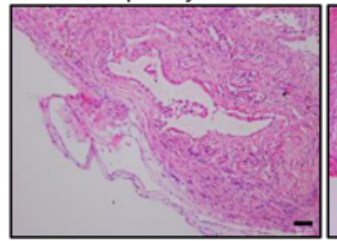

Seminal vesicle

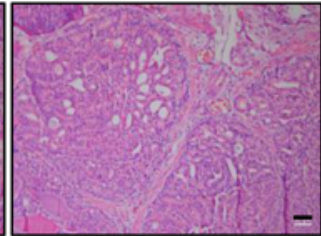

Vas deferens

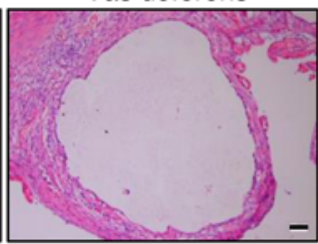

C
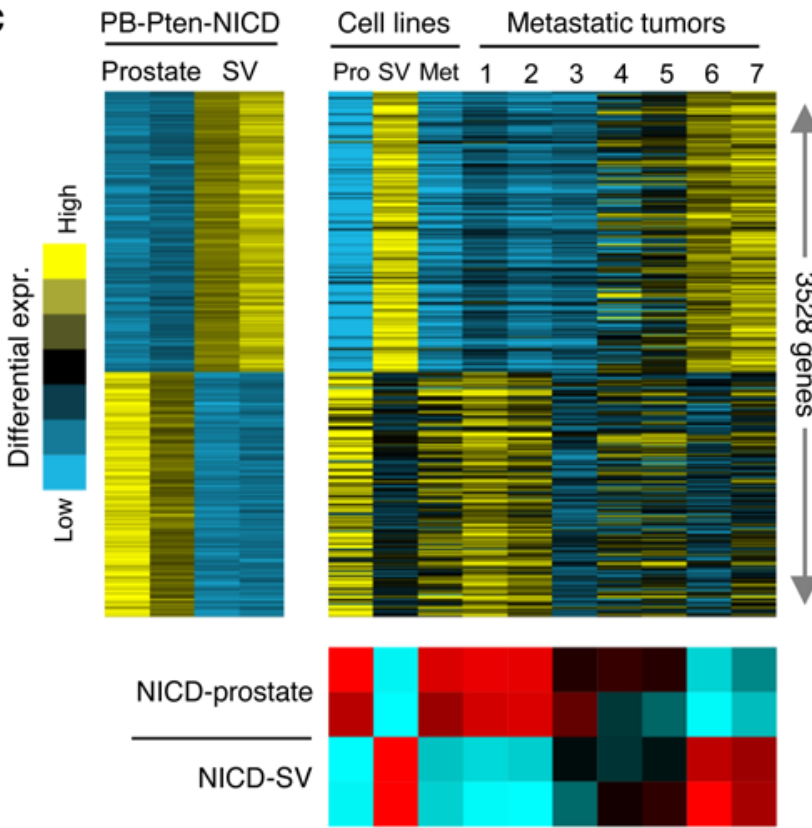

Inter-profile correlation $(r)$

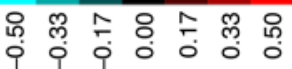

D

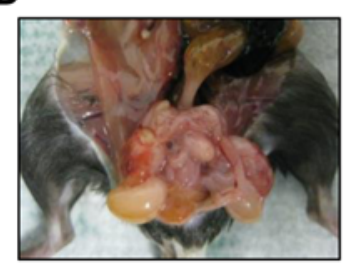

E

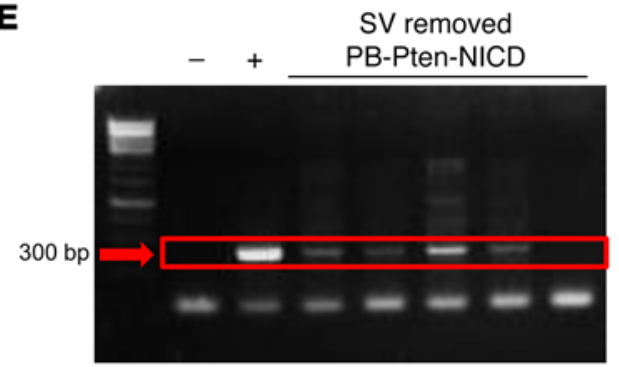

$\mathbf{F}$

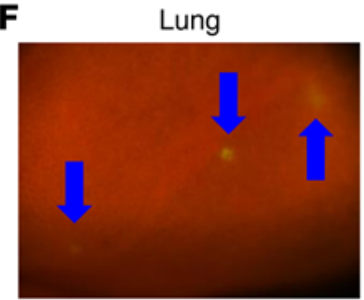

$\mathrm{H} \& \mathrm{E}$

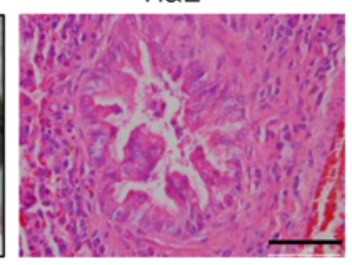

SV removed

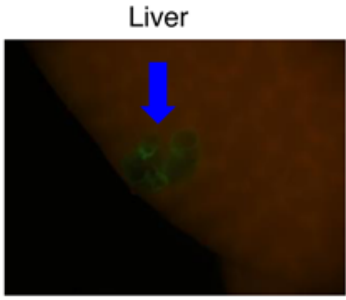

Figure 7. The prostate serves as a tissue of origin for lung metastases in the PB-Pten-NICD model. (A) Image of xenografts outgrown from tissue fragments of prostates, seminal vesicles, epididymides, and vasa deferentia of 4-week-old PB-Pten-NICD mice. Bar graphs show means \pm SD of tissue weight. (B) H\&E staining of xenografts. (C) Heat map on the left shows 3,528 genes differentially expressed in prostate versus seminal vesicles (SVs) (fold 1.4 each prostate profile vs. each SV profile). Heat map on the right shows expression of these genes with the same gene ordering in 7 lung metastases and 3 cell lines derived from PB-Pten-NICD mice. Red-blue heat map shows intersample profile correlations based on the 3,528 genes (by Pearson's coefficient; red, positive). (D) Left shows absence of seminal vesicles in a mouse that has undergone surgical removal of seminal vesicle. H\&E staining (right) shows histology of prostate tissue from this mouse. (E) PCR analysis of Pten deletion allele in circulating blood cells of PB-Pten-NICD mice that have undergone seminal vesicle resection. (F) Fluorescent images of lung and liver tissues from a 34-week-old PB-Pten-NICD-mTmG mouse that has undergone surgical removal of seminal vesicles at 4 weeks of age. Blue arrows denote GFP-positive tumor foci. Scale bars: $50 \mu \mathrm{m}$.

ter referred to as PB-Pten-NICD-mTmG mice). Figure 6, D and E, shows representative images of IHC analyses of the lineage markers in the lung and liver metastases. GFP-expressing metastatic prostate cancer cells in the lung and liver express pAKT, indicating that they were derived from Pten-null cells. Lung metastases are composed of cells that express both CK5 and CK8 or only CK8. AR is expressed in the lung metastases in 16 of 17 mice. In contrast, all metastatic tumor cells in the liver express only CK8, and most of them do not express AR. Bone tissues from mice in both groups were also examined extensively by $\mathrm{H} \& \mathrm{E}$ and IHC analyses, but no lesions in bones were definitively identified by staining with CK8 or AR (data not shown).

The tissue origin of metastatic tumors in the PB-Pten-NICD model. Seminal vesicles, vasa deferentia, and epididymides sel- 
A

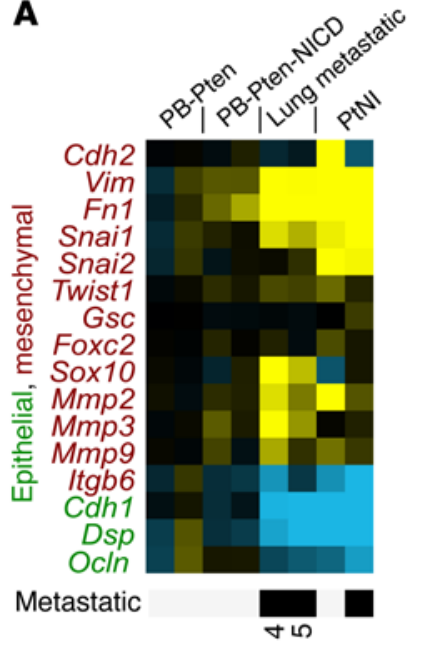

Expr. fold change $1 / 4 \square\left[\begin{array}{ll}1 \\ \square\end{array}\right.$
B

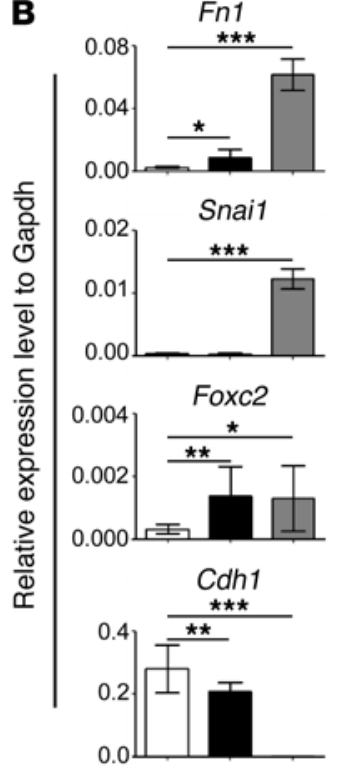

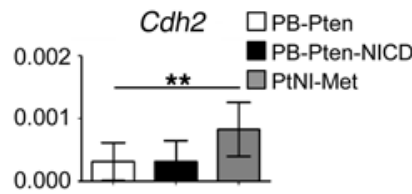
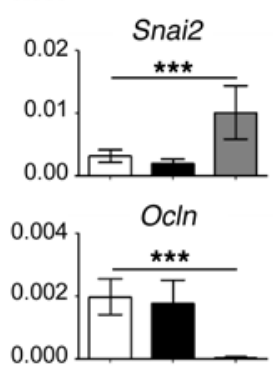

DSP

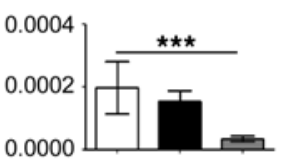

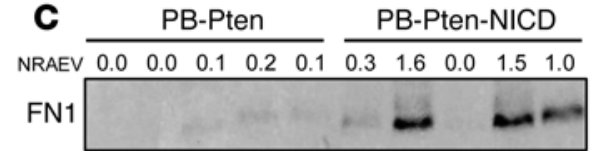

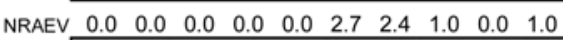

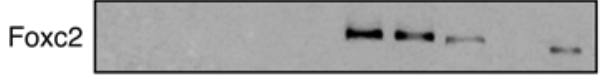

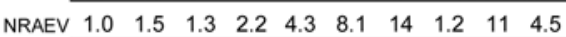

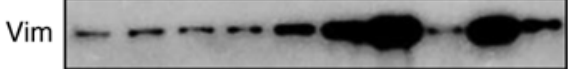

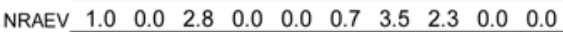

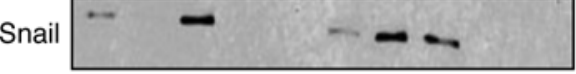

$\begin{array}{lllllllllll}\text { NRAEV } & 0.3 & 0.0 & 1.8 & 4.4 & 0.0 & 0.0 & 3.0 & 0.0 & 2.1 & 1.0\end{array}$

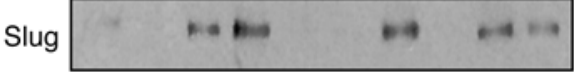

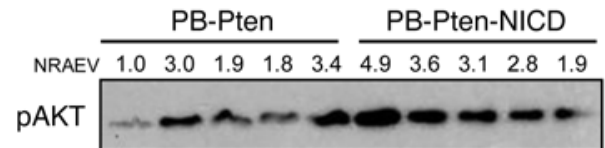

$\begin{array}{lllllllllll}\text { NRAEV } & 1.0 & 0.9 & 1.2 & 1.9 & 0.8 & 1.0 & 0.8 & 0.5 & 0.3 & 0.1\end{array}$

AKT

$\begin{array}{lllllllllll}\text { NRAEV } & 1.0 & 0.6 & 6.8 & 4.5 & 0.4 & 5.3 & 8.4 & 6.2 & 7.7 & 11\end{array}$

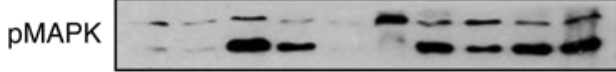

$\begin{array}{lllllllllll}\text { NRAEV } & 1.0 & 0.9 & 1.0 & 1.1 & 1.2 & 1.0 & 1.1 & 0.8 & 0.2 & 0.4\end{array}$

MAPK

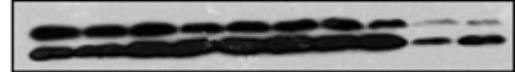

$\beta$-Actin

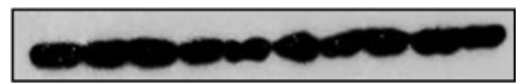

Figure 8. Notch promotes epithelialmesenchymal transition. (A) Heat map of expression of EMT-related genes in prostate tissue of PB-Pten mice (PB-Pten) and PB-Pten-NICD mice (PB-Pten-NICD), 2 prostatederived lung metastases (lung metastatic) from PB-Pten-NICD mice (nos. 4 and 5 in Figure 7C), and 2 cell lines (PtNI) established from prostate primary and metastatic prostate tumors of PB-Pten-NICD mice. (B) qRT-PCR analysis confirms differential expression of 8 genes. Bar graphs show means \pm SD of gene expression in prostate tissues of PB-Pten and PB-Pten-NICD mice ( $n=12$ each), and the PtNI-Met cell line established from metastatic prostate tumors in PB-Pten-NICD mice (9 independent assays on the same cell line). ${ }^{*} P<0.05,{ }^{*} P<0.01,{ }^{* * *} P<0.001$ by ANOVA with Dunnett's correction for multiple comparisons. (C) Western blot analysis of fibronectin (FN1), FOXC2, vimentin (Vim), Snail, Slug, $A K T$, and MAPK in prostate lysates of 16-week-old PB-Pten and PB-PtenNICD mice. Each lane represents an independent specimen. NRAEV, normalized relative arbitrary expression value by $\beta$-actin. (D) Coimmunostaining of vimentin and $\alpha$-smooth muscle actin (SMa) in prostate tissues of 16-week-old PB-Pten and PB-PtenNICD mice. Scale bars: $50 \mu \mathrm{m}$.

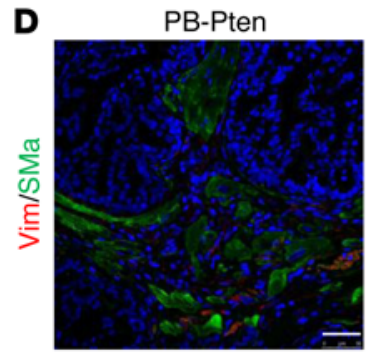

\section{PB-Pten-NICD}

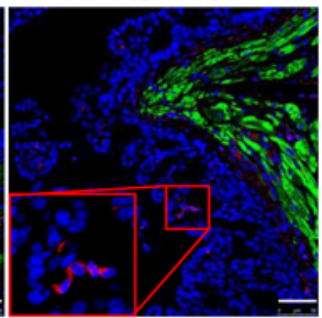

dom transform in mouse models for prostate cancer or in humans. However, all these organs are consistently enlarged in PB-PtenNICD mice. Organ enlargement may reflect growth of dichotomous tumors, or growth of tumor cells migrated from the prostate, or accumulation of secretory fluids due to outlet obstruction. To distinguish these possibilities, we dissected tissue fragments of equal size from these organs and prostate tissues from 4 -week-old PB-Pten-NICD mice and transplanted them under the kidney capsules of immunodeficient male host mice. At 4 weeks of age, all these organs were morphologically normal, as the probasin promoter was just activated. Figure 7, A and B, shows that transplanted seminal vesicles and prostate tissues propagated extensively in
4 weeks and formed tumors that were histologically reminiscent of those formed in situ. In contrast, xenografts of vasa deferentia and epididymides displayed little growth and also were histologically reminiscent of their respective normal organs. These observations indicate that both prostate and seminal vesicles are capable of developing dichotomous tumors in the PB-Pten-NICD model, and raised the question of the identity of the tissue of origin for the distal metastases.

To investigate the identity of the tissue of origin for the metastases, we compared gene expression profiles of the prostate tissues and seminal vesicles from PB-Pten-NICD mice and those of 7 lung metastases from different mice. We established 3 cell lines 
A RBPJ binding motif: TGGGAA or TGAGAA $-2653-2399$

\begin{tabular}{|l|l|l|l|}
\hline & & & mFoxc2 \\
\hline
\end{tabular}
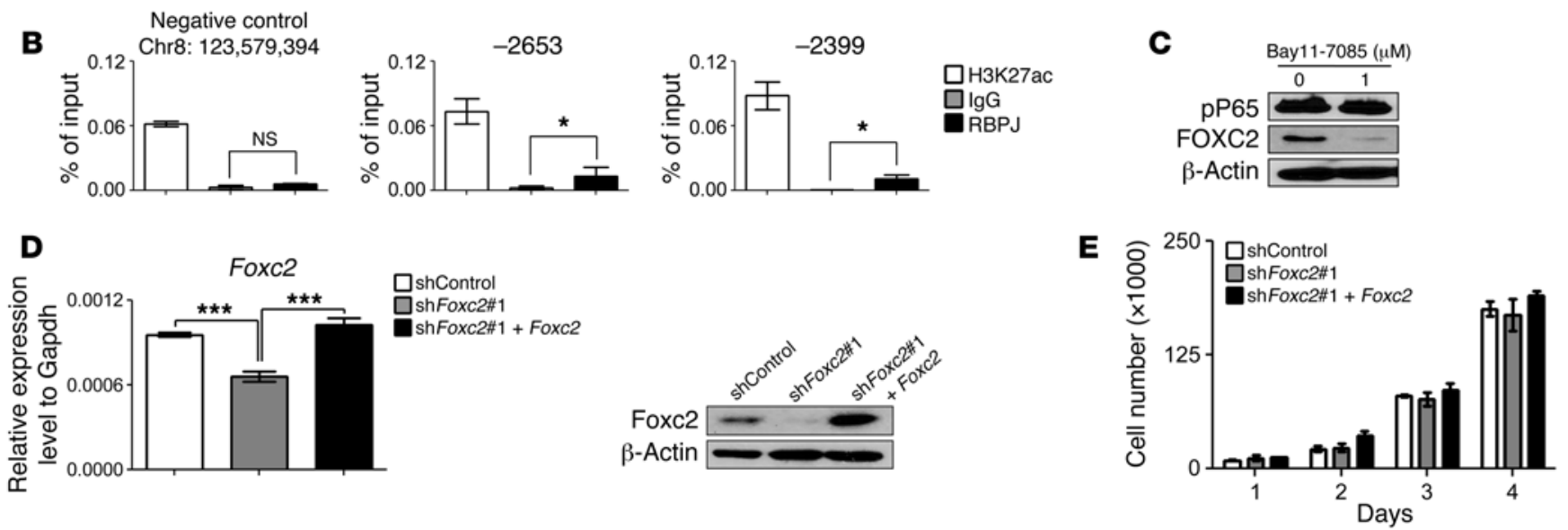

C

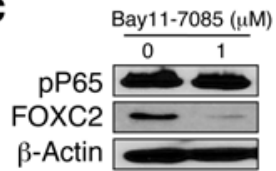

shFoxc2\#1

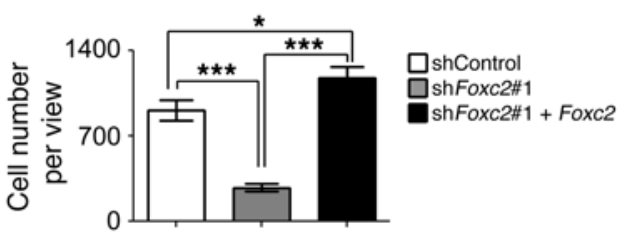

G
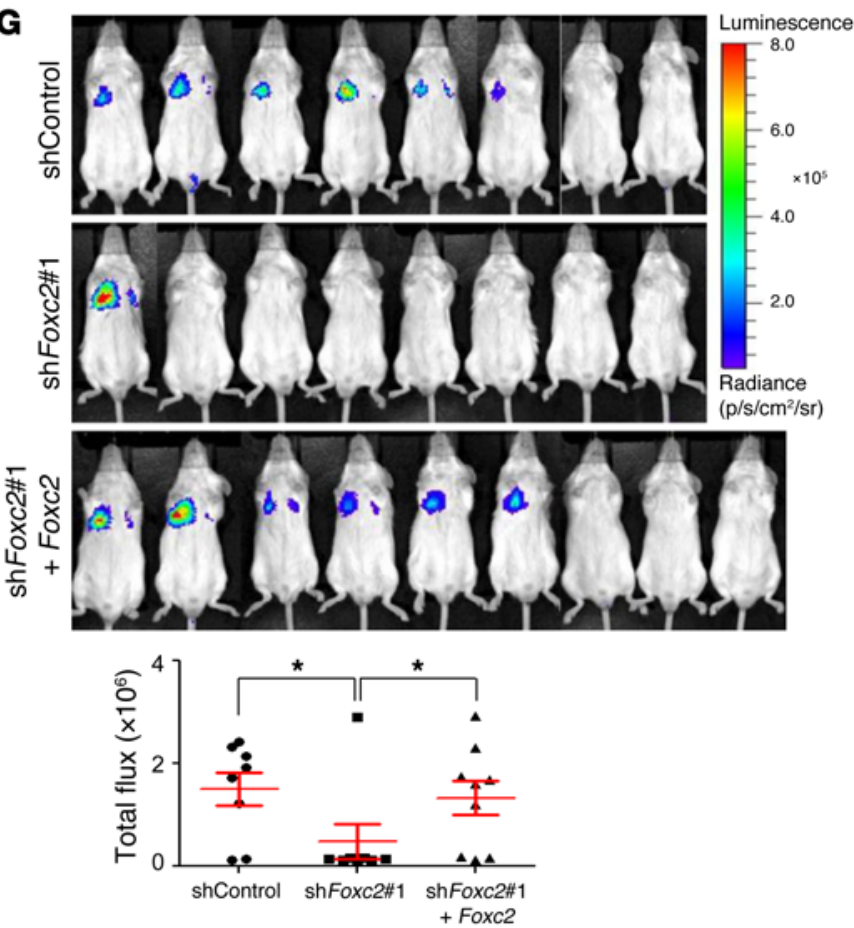
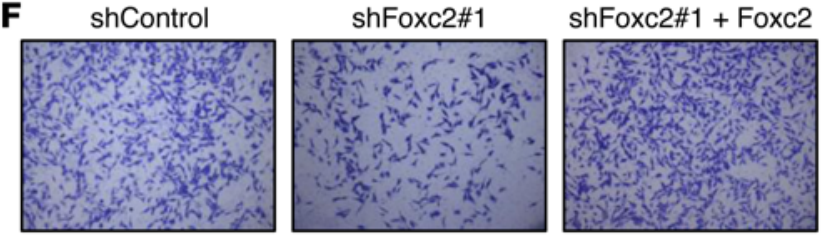

Figure 9. Notch promotes tumor metastasis by regulating FOXC2. (A) Schematic illustration of RBPJ binding sites in Foxc2 promoter. (B) ChIP assay for RBPJ and H3K27ac using the PB-Pten-NICD metastatic cell line. ${ }^{*} P<0.05$ by Student's $t$ test. (C) Western blot analysis of phospho-P65 and F0XC2 in PBPten-NICD metastatic cell line with and without Bay11-7085 treatment. (D) qRT-PCR and Western blot analyses of PB-Pten-NICD metastatic cells infected with scrambled shRNA lentivirus, Foxc2 shRNA\#1 lentivirus, and both. ${ }^{* *} P<0.001$ by ANOVA Bonferroni post hoc test. (E) Growth curves of PB-PtenNICD metastatic cells infected with scrambled shRNA lentivirus, Foxc2 shRNA\#1 lentivirus, and both. No significant difference is detected among groups by 2-way ANOVA. (F) Transwell migration assay. Bar graphs show means $\pm \mathrm{SD}$ of migrated cells per well from 3 independent experiments. ${ }^{*} P<0.05$, ${ }^{* * *} P<0.001$ by ANOVA Bonferroni post hoc test. (C) NOD/SCID mice were inoculated with $2 \times 10^{6}$ cells each in individual groups via tail vein. Mice were imaged 5 weeks later. Bar graphs show means \pm SD of bioluminescent signals. ${ }^{*} P<0.05$ by ANOVA Bonferroni post hoc test.

from primary seminal vesicle tumors, primary prostate tumors, and lung metastases in PB-Pten-NICD mice, respectively (Supplemental Figure 6A). These cell lines were also included in the microarray analysis. We hypothesized that the gene expression profiles of established cell lines and metastatic tumors should resemble those of their primary tumors of origin. Of approximately 60,000 probes, we identified 3,528 probes with at least 1.4 -fold change between the seminal vesicles and prostate tumors (Figure 7C and Supplemental Figure 7). Consistent with our hypothesis, the expression profiles of the 2 cell lines established from primary tumors resemble those of their respective tissues of origin. The expression profile of the metastatic cell line resembles those of the prostate tumor tissues, indicating the prostate as its tissue of origin. This is further confirmed by qRT-PCR analysis show- 

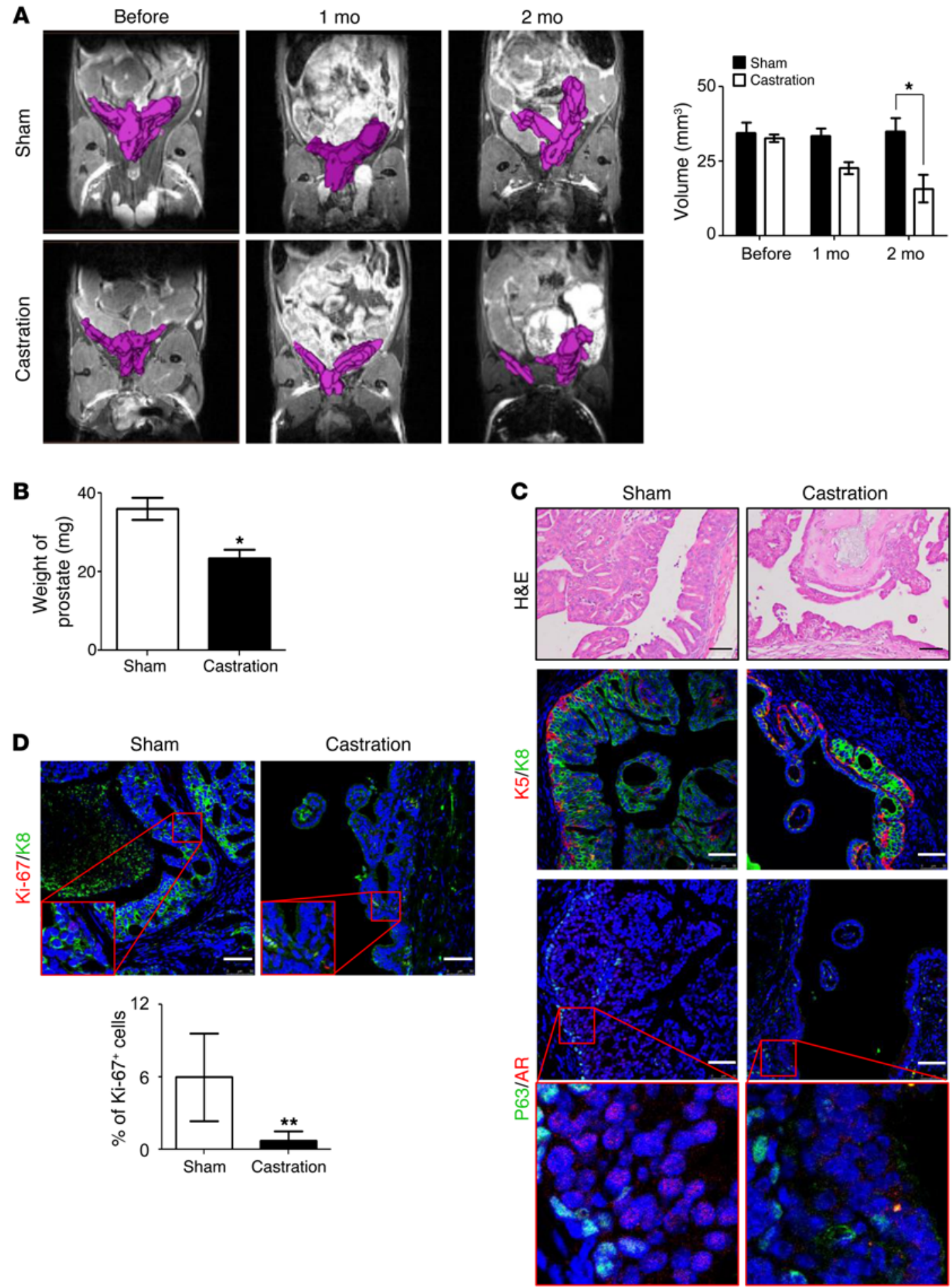

Figure 10. Notch activation does not confer capacity for castration resistance. (A) Magnetic resonance imaging of PB-Pten-NICD prostate tissues before and after castration. Bar graph shows means \pm SD of prostate volume based on MRI analysis. $n=3$. (B) Bar graph shows prostate weight of mice 2 months after castration or sham surgery. $n=3$. (C) H\&E staining (top), coimmunostaining of K5 and K8 (middle), and P63 and AR (bottom) in prostate tissues of PB-Pten-NICD mice that underwent castration and sham surgery. Scale bars: $50 \mu \mathrm{m}$. (D) Coimmunostaining of Ki-67 and K8 in prostate tissues of PB-PtenNICD mice that underwent castration and sham surgery. Scale bars: $50 \mu \mathrm{m}$. Bar graphs show means $\pm \mathrm{SD}$. ${ }^{*} P<0.05$, ${ }^{* *} P<0.01$ by Student's $t$ test.

ing that several genes expressed at high levels in murine seminal vesicles (32) were poorly expressed in this cell line and the cell line derived from primary prostate tumor (Supplemental Figure $6 \mathrm{~B})$. Figure $7 \mathrm{C}$ also shows that the expression profiles of each of the 2 metastatic specimens resemble those of the seminal vesicle tumors and the prostate tumors, respectively, while the expression profiles of the remaining 3 metastatic specimens display a mixed pattern. This study suggests that metastatic tumors in this 
model may have originated from either the prostate or the seminal vesicles, or both.

To further directly demonstrate that prostate tumor cells are capable of metastasizing in this model, we removed the seminal vesicles in 4-week-old experimental PB-Pten-NICD-mTmG mice when they were still morphologically normal. Three of seven mice did not have visible outgrowth of seminal vesicle tissues 32 weeks later, indicating the complete removal of seminal vesicles (Figure 7D). However, circulating tumor cells in the blood were readily detectable by PCR analysis (Figure 7E), and micrometastases were observed in the lung and liver in these 3 mice (arrows, Figure 7F). This study directly demonstrates that Notch signaling is capable of promoting metastasis of Pten-null prostate cancer cells.

FOXC2 is critical for Notch-mediated prostate cancer metastasis. Gene expression array analysis revealed that the expression levels of several genes related to epithelial-mesenchymal transition (EMT) were altered consistent with an EMT process in the primary and metastatic tumor tissues and the established prostate cancer cell lines from PB-Pten-NICD mice, as compared with those of the PB-Pten primary prostate tumors (Figure 8A). qRT-PCR analysis confirmed some of these results (Figure 8B). Figure 8C further shows that fibronectin, FOXC2, and vimentin were also upregulated at the protein level in PB-Pten-NICD mouse prostates, whereas the changes in expression of Snail and Slug were not consistent. Western blot analysis also reveals increased AKT and MAPK activities in the PB-Pten-NICD group. IHC analysis further showed that vimentin is expressed by small patches of epithelial cells in PB-Pten-NICD prostate tissues (Figure 8D). These results suggest that Notch signaling may promote invasion and metastasis by promoting EMT.

FOXC2 plays a critical role in regulating breast cancer metastasis (33) and has been shown to be upregulated in other metastatic prostate cancer models (34). It can be induced by a variety of EMTinducing agents, including Snail and Twist, suggesting it is commonly involved in a diverse set of EMT programs (35). In addition, FOXC2 was significantly upregulated at both the mRNA and the protein level in the prostate tissues of PB-Pten-NICD mice, whereas other EMT mediators such as Snail and Slug were not as consistently upregulated at the protein level (Figure 8C). Finally, Notch was recently shown to upregulate Foxc2 to promote definitive hematopoiesis (36). Therefore, we reasoned that FOXC2 may play a critical role in tumor metastasis induced by elevated Notch activity.

There are 2 putative RBPJ binding elements (TGGGAA or TGAGAA) at the promoter region of Foxc2 (Figure 9A). Raw data from a previous genome-wide ChIP sequencing analysis by Castel et al. indicate that RBPJ may directly bind to these 2 loci (37). We confirmed that RBPJ directly bound to these 2 loci by performing a ChIP assay using the metastatic prostate cancer cell line established from PB-Pten-NICD mice (Figure 9B). In contrast, RBPJ was not enriched at a negative control locus where RBPJ should not bind according to Castel et al. (37). In addition, H3K27ac, a histone modification mark for active transcription, was also enriched in the RBPJ binding regions. These results suggest that Notch is capable of positively regulating Foxc2 directly. On the other hand, Foxc2 has been shown to be directly regulated by NFKB (38). We showed previously that Notch signaling augments the NFKB activity in prostate epithelial cells (17), which is further corroborated by
Western blot analysis of P65 phosphorylation in prostate lysates of PB-Pten and PB-Pten-NICD mice (Supplemental Figure 8). Suppressing the NFKB signaling using its inhibitor Bay11-7085 inhibited viability and in vitro migratory capacity of the PB-Pten-NICD metastatic prostate cancer cell line (Supplemental Figure 9). Figure 9C shows that FOXC2 was significantly downregulated in the PB-Pten-NICD cells even when cells were treated with Bay11-7085 at a dosage that did not substantially affect cell viability. Collectively, these results show that Notch is capable of inducing Foxc2 expression both directly and indirectly. The efficient suppression of Foxc 2 by Bay11-7085 suggests the indirect regulation of Foxc 2 by Notch via NFKB as a major mechanism.

To determine whether FOXC2 plays a critical role in Notchmediated prostate cancer metastasis, we examined whether suppressing FOXC2 in the PB-Pten-NICD metastatic prostate cancer cell line would inhibit its metastatic potential. Figure 9D and Supplemental Figure 10A show that Foxc2 was downregulated efficiently by 2 independent shRNAs. Foxc 2 suppression did not affect cell proliferation (Figure 9E and Supplemental Figure 10B), but suppressed the capability of the PB-Pten-NICD metastatic cells of invading into Matrigel in the in vitro chamber assay (Figure 9F and Supplemental Figure 10C) and reduced their capacity to form distal metastases in the lung when injected into the tail veins of NOD/SCID mice (Figure $9 \mathrm{G}$ and Supplemental Figure 10D). Reintroducing a shRNAresistant Foxc2 into the cells abolished the suppression of in vitro migration and in vivo metastasis mediated by the shRNA (Figure 9, F and G), corroborating the specificity of the Foxc2 shRNA. These studies demonstrate FOXC2 as a major downstream mediator for Notch-induced prostate cancer metastasis.

PB-Pten-NICD tumor cells respond to androgen deprivation. We sought to determine whether Notch activation promotes castration-resistant prostate cancer. Briefly, 12-week-old PB-PtenNICD mice either were castrated or underwent mock surgeries as the controls. MRI analyses were performed to measure tumor volume before castration and 1 month and 2 months after castration. MRI showed that prostate tumor decreased in size by $45 \%$ two months after castration (Figure 10A), which is corroborated by the prostate weights (Figure 10B). IHC analysis reveals a reduction of CK8-expressing luminal cells after androgen ablation (Figure 10C). Figure 10C also shows that AR is localized in the nucleus of prostate tumor cells in the control group, but was exported into the cytoplasm after castration, corroborating successful androgen ablation. Ki-67 staining also shows that the proliferative index was reduced by $11.8 \%$ after castration (Figure 10D). These results indicate that Notch signaling is insufficient to confer the capacity for castration resistance in the Pten-null prostate cancer model.

\section{Discussion}

A multifaceted role of Notch signaling in prostate cancer progression. Notch can act as either an oncogene or a tumor suppressor in different tumor models. For example, a definitive role of Notch as an oncogene has been clearly demonstrated in T cell acute lymphoblastic leukemia (5). In contrast, Notch signaling displays tumorsuppressive features in myeloid leukemia and skin and bladder cancers (39-41). The functions of opposite effects of Notch signaling can even be observed in distinct tumor subtypes originated from the same organ $(5,40)$. These reports highlight a multifacet- 
ed role of Notch signaling in different cellular contexts, which has been partially explained by cross-talk between Notch and other tissue-specific master regulators (4).

The expression of Notch pathway elements in established cancer cell lines, transgenic mouse models, and clinical tumor specimens unambiguously indicates a deregulation of the Notch signaling pathway in prostate cancer (7-9). However, functional studies in human cancer cells by modulation of Notch pathway components suggest either negative (18), positive $(14,15,42)$, or neutral (16) impact of Notch on prostate cancer cell proliferation and apoptosis in vitro. These controversies may be explained as follows: First, the dosage and extent of Notch signaling are critical in dictating biological outcomes. It has been shown that a low level of Notch activity promoted proliferation of MCF-10A cells in a $3 \mathrm{D}$ culture while higher levels of Notch activity suppressed their growth (43). Second, Notch-mediated biology is also dependent on specific genetic contexts. For example, Notch is capable of suppressing Pten expression through HES1 (25). Therefore, Notch activation in Pten-intact and Pten-null tumor cells may result in different biological outcomes (44). Finally, different Notch ligands and receptors may mediate distinct or even opposite biological outcomes $(45,46)$, and expression of individual ligands or receptors does not guarantee signaling activation (47).

Given the aforementioned diverse regulatory mechanisms, it is not unexpected that augmented Notch activity in the Ptennull model promotes apoptosis and causes a reduction in primary tumor burden. The observed increase in DNA damage in the PBPten-NICD mouse prostates may have resulted from stress signals induced by uncontrolled cellular proliferation. Excessive DNA damage induces p53-mediated apoptosis. p53 has been shown previously to serve as a barrier for progression of Pten-null prostate tumor by inducing cellular senescence (48). Future studies are necessary to determine whether senescence also plays a role in the suppressed primary tumor growth in the PB-Pten-NICD model. In addition, Dail et al. showed previously that inhibition of the PI3K activity in T cell leukemia selected for resistant cells that possessed decreased Notch activity (49). This study suggests that Notch suppresses PI3K activity through yet-to-be-defined mechanisms. Although our study shows a mild increase in AKT activation in prostate tissues of PB-Pten-NICD mice, Notch may affect the activity of upstream kinases, which in turn impairs cell survival independent of AKT.

On the other hand, most in vitro studies consistently support that Notch signaling promotes several malignant features of prostate cancer cells, such as migration and invasion (50), osteomimetic properties (11), chemoresistance (13), and stemness (12). Our in vivo genetic study demonstrates that endogenous epithelial Notch signaling is not essential for tumor initiation and progression in the Pten-null model. However, augmented Notch activity is capable of promoting tumor cell metastasis. Our study suggests that Notch promotes metastatic prostate cancer by promoting EMT and that FOXC2 is necessary for Notch-mediated tumor cell metastasis. FOXC2 may promote tumor metastasis in the PB-Pten-NCID model by activating the PDGFR/MAPK kinase signaling axis (Figure $8 C)(33,51)$, as previous studies have shown that synergy between PI3K and MAPK signaling actively promotes metastasis $(34,52)$. Collectively, these in vitro and in vivo studies suggest that Notch may serve as a promising therapeutic target for metastatic and chemoresistant prostate cancers, whereas targeting Notch may not suppress primary tumor growth necessarily. A very recent study has demonstrated that the $\gamma$-secretase inhibitor PF-03084014 enhances the therapeutic efficacy of docetaxol in prostate cancer xenograft models (53). It should be noted that recent studies indicated that EMT is dispensable for tumor metastasis $(54,55)$. We showed that NFKB sits upstream of FOXC2 and is critical for both the survival and the migratory capacity of PBPten-NICD cells (Supplemental Figure 9). Therefore, Notch may also promote metastasis via other NFKB-mediated FOXC2-independent mechanisms (17).

Nevertheless, because of our inadequate understanding of the role of Notch in prostate cancer, caveats persist when the Notch signaling pathway is targeted clinically. Additional preclinical studies should be performed to determine how Notch affects prostate cancer progression using different mouse models induced by distinct oncogenic signaling. In addition, the recently established prostate organoid assays $(56,57)$ may be applied to establish correlations between genomic landscapes and the response to Notch inhibition in a rapid and cost-effective manner. These future studies will help determine whether and how Notch should be employed as a therapeutic target.

Transformation of seminal vesicle epithelial cells in the PB-PtenNICD model. An unexpected finding in our study is that seminal vesicles were transformed in this model. The prostate gland and seminal vesicles are the 2 major male sex-accessory glands in mammals. They share many histological and functional features (58). Interestingly, the incidence of cancer in these 2 organs is drastically different. Prostate cancer is the second leading cause of cancer-related death in men in the United States (59), whereas very few cases of adenocarcinoma of seminal vesicles have been reported in the literature (60). This observation also holds true in the mouse models. During the past 2 decades, a dozen mouse models for prostate cancer have been generated in which oncogenic signaling is often activated via the probasin promoter. Interestingly, although the probasin promoter is also active in seminal vesicles, transformation of seminal vesicles is rarely observed in these models. The distinct incidence of cancer in these 2 organs probably reflects the differences of their inherent properties shaped by their different embryonic origins. Developmentally, the seminal vesicles have a mesodermal origin, while the prostate is mainly derived from the endodermal layer (61). Thus, they may use different sets of master transcription factors to maintain their lineage identities and differentiated states. As Notch signaling has been shown to play a critical role in regulating lineage commitment and cell fate determination (2), it is possible that enhanced Notch signaling interferes with the activities of the seminal vesicle-specific master transcription factors and alters the differentiation states of seminal vesicle epithelial cells, thereby sensitizing them to the transformation induced by loss of function of PTEN.

\section{Methods}

Mice and genotyping. The C57BL/6 and NOD/SCID mice were purchased

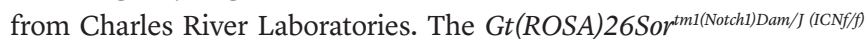
mice were purchased from The Jackson Laboratory. The Pten ${ }^{f / f l}$ mice were from Hong Wu at UCLA (Los Angeles, California, USA) (26). The 
ARR2PB-Cre transgenic mice were from Fen Wang at the Institute of Bioscience and Technology, Texas A\&M Health Science Center (Round Rock, TX). The Rbpj $j^{f / f l}$ mice were from Tasuku Honjo at Kyoto University (Kyoto, Japan). The mTmG fluorescence reporter mouse line was generated by the group of Liqun Luo at Stanford University (Stanford, California) (31). Mice were genotyped by PCR using mouse genomic DNA from tail biopsy specimens. The sequences of genotyping primers are listed in Supplemental Table 1. PCR products were separated electrophoretically on $1 \%$ agarose gels and visualized via ethidium bromide under UV light. Experimental mice were castrated at the age of 8 weeks using standard techniques as described previously (62). Seminal vesicles were removed by grabbing of the bottom of the seminal vesicles near the urethra with fine dissecting forceps.

Establishing cell lines from prostate tumors and lung metastases. Primary and metastatic tumor tissues were cut into small pieces of approximately $1 \mathrm{~mm}^{3}$. Tissues were digested in DMEM/collagenase/ hyaluronidase/FBS (Invitrogen) for 1 hour at $37^{\circ} \mathrm{C}$, followed by an additional 1 hour of digestion in $0.25 \%$ trypsin-EDTA (Invitrogen) on ice. Cells were then passed through $70-\mu \mathrm{m}$ cell strainers (BD Biosciences) to get single cells. Dissociated single cells were cultured in $10 \%$ FBS DMEM culture medium, including $6 \mathrm{ng} / \mathrm{ml}$ EGF (Invitrogen) and $5 \mu \mathrm{g} / \mathrm{ml}$ insulin (Invitrogen). Because epithelial cells in the PB-Pten-NICD model specifically express a low level of GFP driven by the ROSA26 locus, epithelial cells were further FACS-purified from the outgrown cell mixtures. All cell lines were maintained at $37^{\circ} \mathrm{C}$ in a humid atmosphere containing $5 \% \mathrm{CO}_{2}$.

In vitro and in vivo cellular assays. For in vitro proliferation assay, $2 \times 10^{3}$ cells were seeded in 96-well plates. Cells were counted for 3 sequential days. The in vitro cell migration assay was performed using the BioCoat Matrigel Invasion Chamber (BD Biosciences). Briefly, $2 \times 10^{4}$ cells were put in each chamber and incubated for 24 hours before invaded cells were quantified according to the manufacturer's instruction.

Pharmacological manipulation of NFKB signaling. BAY11-7085 was purchased from Tocris Bioscience. Compounds were dissolved in DMSO and used in vitro at a concentration indicated in Figure 9C and Supplemental Figure 9. Cell culture medium was replaced every 24 hours.

Foxc2 overexpression and suppression in the PB-Pten-NICD cells. A Foxc2 shRNA (sc-45366-v) and the scrambled control (sc-45366-sh) lentiviruses were purchased from Santa Cruz Biotechnology. The results from this shRNA are presented in Supplemental Figure 10. The results obtained from the other Foxc2 shRNA targeting the Foxc2 3'-UTR region (TRC0000321411; Sigma-Aldrich) are presented in Figure 9. The mouse Foxc2 cDNA was obtained from Sendurai Mani and was cloned into the FU-CGW vector (63) by a blunt-end ligation into a filled-in EcoRI site, resulting in the FU-Foxc2-CGW vector. The PB-Pten-NICD metastatic cells were infected with FU-LuciferaseCRW lentivirus, and RFP-expressing cells were FACS sorted. Cells were infected with shRNA lentivirus or the FU-Foxc2-CGW lentivirus at an MOI of 10 and selected with puromycin or FACS-sorted based on GFP expression.

Bioluminescence imaging. $2 \times 10^{6}$ PB-Pten-NICD metastasis cells in $150 \mu \mathrm{l}$ PBS were injected into 10-week-old male NOD/SCID mice through the tail vein. Animals were imaged weekly using an IVIS Lumina II (Advanced Molecular Vision) following the manufacturer's recommended procedures and settings.

Detection of circulating tumor cells in blood. Blood samples were harvested through a heart puncher, and genomic DNA was isolated using the
PureLink Genomic DNA Mini Kit (Invitrogen) according to the manufacturer's instructions. Pten-null cells were detected by PCR using $50 \mathrm{ng}$ of genomic DNA template with forward primer 5'-ACTCAAGGCAGGGATGAGC-3' and reverse primer 5'-GCTTGATATCGAATTCCTGCAGC-3'. Final products were electrophoretically separated on agarose gels and visualized via ethidium bromide under UV light.

MRI analysis. MRI images in this study were acquired with a 9.4T Bruker Avance Biospec Spectrometer 21-cm-bore horizontal scanner with a $35-\mathrm{mm}$ volume resonator (Bruker BioSpin). Mice were anesthetized with $5 \%$ isoflurane and then maintained on $2 \%$ isoflurane in $100 \% \mathrm{O}_{2}$ during imaging. During imaging, respiratory rates were monitored and temperature was maintained at $37^{\circ} \mathrm{C}$ using an air heating system with model 1025 Small Animal Monitoring and Gating System Software (SA Instruments Inc.). Anatomical images were acquired using a 3D TurburRARE sequence with ParaVision 5.0 (Bruker BioSpin). The field of view was $3.0 \mathrm{~cm} \times 3.0 \mathrm{~cm} \times 3.0 \mathrm{~cm}$; the repetition time was 2,000 ms; the echo time was $10 \mathrm{~ms}$; the rapid acquisition with relaxation enhancement (RARE) factor was 8; the flip angle was $180.0^{\circ}$; the number of averages was set to 1 ; and the matrix size was $128 \times 128 \times 128$. Tumor and prostate volumes were analyzed using AMIRA software (FEI).

RNA isolation and qRT-PCR. Total RNA was isolated from cells using the RNeasy Plus Mini Kit (Qiagen). Reverse transcription was performed using the iScript cDNA synthesis kit (Bio-Rad). qRT-PCR was performed using the SYBR Premix Ex Taq (Perfect Real Time; Takara Bio Inc.) on a StepOnePlus Real-Time PCR system (Applied Biosystems). Primer sequences for qPCR are listed in Supplemental Table 2.

Western blot. Prostate tissues were lysed in RIPA buffer (20 mM Tris-HCl, pH 7.5, $150 \mathrm{mM} \mathrm{NaCl}, 1$ mM Na${ }_{2}$ EDTA, 1 mM EGTA, 1\% NP-40, $1 \%$ sodium deoxycholate, $2.5 \mathrm{mM}$ sodium pyrophosphate, $1 \mathrm{mM} \beta$-glycerophosphate, $1 \mathrm{mM} \mathrm{Na} \mathrm{VO}_{4}$ ) with protease inhibitors and phosphatase inhibitors (Roche Applied Science) using Tissuelyser LT (Qiagen). Protein concentrations were determined by a Bradford Assay kit (Bio-Rad). Protein was separated by $8 \%$ or $12 \%$ SDS-PAGE and transferred onto a PVDF membrane (Amersham Biosciences). The membrane was blocked in $5 \%$ skim milk, subsequently incubated with primary antibodies listed in Supplemental Table 3 at $4^{\circ} \mathrm{C}$ overnight followed by incubation with peroxidase-conjugated goat antimouse IgG or goat anti-rabbit IgG (Jackson ImmunoResearch Inc.), and developed with Pierce ECL reagent (Thermo Scientific). Results were quantified using Image $(\mathrm{NIH})$.

Chromatin immunoprecipitation. ChIP assays were performed using the ChIP-IT Express kit (Active Motif) following the manufacturer's protocol except for the DNA purification step. The QIAquick PCR purification kit was used for DNA purification after anti-crosslink of immunoprecipitated DNA. Purified DNA was eluted by 50 $\mu \mathrm{l}$ of elution buffer, and $3 \mu \mathrm{l}$ was used for quantitative PCR analysis using the SensiFAST SYBR Hi-ROX kit (Bioline). The negative control site is located at Chr8:123,578,394-123,578,470. The primers for amplification of targeting sites are: negative control forward 5'-CCTGATGCGTGGTACACTTG-3', negative control reverse 5'-AAGGCAGGACCCAGAAAAAC-3', RBPJ binding site 2653 forward 5'-GTGGGTCGATCCATTTGAGA-3', RBPJ binding site 2653 reverse 5'-GCGTATGCCAAATTTAAACCA-3', RBPJ binding site 2399 forward 5'-TGGAATTTTCAGCAACATCG-3', RBPJ binding site 2399 reverse 5'-GAAAACGTCTTGCCCCTCAT-3'. The antibodies used in ChIP assays were IgG (sc-2025; Santa Cruz Biotechnology), H3K27ac 
(ab4729; Abcam), and RBPJ (D10A4; Cell Signaling Technology). For each ChIP, $3 \mu \mathrm{g}$ of antibody was used.

Histology and immunostaining. H\&E staining and immunofluorescence staining were performed using standard protocols on $5-\mu \mathrm{m}$ paraffin sections. Slides were incubated with $5 \%$ normal goat serum (Vector Laboratories) and with primary antibodies diluted in $2.5 \%$ normal goat serum overnight at $4^{\circ} \mathrm{C}$. Primary antibodies used in this study are listed in Supplemental Table 4. Slides were then incubated with secondary antibodies (diluted 1:500 in PBS with Tween 20 [PBST]) labeled with Alexa Fluor 488, 594, or 633 (Invitrogen/ Molecular Probes). Sections were counterstained with DAPI (SigmaAldrich). Immunofluorescence staining was imaged using an Olympus BX60 fluorescence microscope (Olympus Optical Co. Ltd.) or a Leica EL6000 confocal microscope (Leica Microsystems). Cell number was determined using the count feature in the software, which asks for the user to indicate the color that would be used to indicate a positive cell (for example, blue would be indicated to count nuclei and thus indicate total numbers of cells). Borders were created such that only epithelial cells would be analyzed.

Expression microarray. Expression microarray assays were performed using 4x60K Whole Mouse Genome Oligo Microarray chip (Agilent Technologies), and Feature Extraction Software 9.1.3.1 (Agilent Technologies) was used to extract and analyze the signals. Array data have been deposited in GEO (GSE72549).

Statistics. All experiments were performed using 2-17 mice in independent experiments. Data are presented as mean $\pm \mathrm{SD}$. ANOVA was used to compare among groups, whereas Student's $t$ test was used to determine significance between groups. All $P$ values reported are 2-sided unless otherwise noted. For all statistical tests, the 0.05 level of confidence was accepted for statistical significance. For computing gene signature scores based on expression profiling data from human tumors, genes were first $z$-normalized to SD from the median across the primary tumor samples, and the average of the $z$-normalized values for all the genes in the signature was used to represent the signature score for each sample profile.

Study approval. All animals used in this study received humane care that adheres to principles stated in the Guide for the Care and Use of Laboratory Animals (NIH publication, 1996 edition), and the protocol was approved by the IACUC of Baylor College of Medicine.

\section{Author contributions}

LX and O-JK designed research studies. O-JK, LZ, JW, QS, and $\mathrm{QF}$ conducted experiments and acquired data. LX, O-JK, LZ, $\mathrm{XH}-\mathrm{FZ}, \mathrm{RP}, \mathrm{MMI}$, and CJC analyzed data. SAM provided reagents. LX wrote the manuscript.

\section{Acknowledgments}

We thank Joseph Valdez for initiating some early studies, Boyu Zhang for technical help, Yiqun Zhang for analyzing expression microarray analyses, the Small Animal Imaging Facilities at Baylor College of Medicine and Texas Children's Hospital, the Cytometry and Cell Sorting Core at Baylor College of Medicine for technical support with funding from the NIH (P30-AI036211, P30-CA125123, and S10-RR024574), and Joel M. Sederstrom for expert assistance. This work is supported by NIH R01-CA190378 and R21-CA196570 (to L. Xin), NIH U01-CA141497 (to M.M. Ittmann), and NIH P30-CA125123 (the Cancer Center Shared Resources Grant).

Address correspondence to: Li Xin, M533 DeBakey Building, BCM MS130, Baylor College of Medicine, One Baylor Plaza, Houston, Texas 77030, USA. Phone: 713.798.1650; E-mail: xin@bcm.edu.
1. Kopan R, Ilagan MX. The canonical Notch signaling pathway: unfolding the activation mechanism. Cell. 2009;137(2):216-233.

2. Lai EC. Notch signaling: control of cell communication and cell fate. Development. 2004;131(5):965-973.

3. Radtke F, Raj K. The role of Notch in tumorigenesis: oncogene or tumour suppressor? Nat Rev Cancer. 2003;3(10):756-767.

4. Ranganathan P, Weaver KL, Capobianco AJ. Notch signalling in solid tumours: a little bit of everything but not all the time. Nat Rev Cancer. 2011;11(5):338-351.

5. Weng AP, et al. Activating mutations of NOTCH1 in human T cell acute lymphoblastic leukemia. Science. 2004;306(5694):269-271.

6. Robinson $\mathrm{D}$, et al. Integrative clinical genomics of advanced prostate cancer. Cell. 2015;161(5):1215-1228.

7. Zhu H, Zhou X, Redfield S, Lewin J, Miele L. Elevated Jagged-1 and Notch-1 expression in high grade and metastatic prostate cancers. Am J Transl Res. 2013;5(3):368-378.

8. Santagata S, et al. JAGGED1 expression is associated with prostate cancer metastasis and recurrence. Cancer Res. 2004;64(19):6854-6857.

9. Danza G, et al. Notch3 is activated by chronic hypoxia and contributes to the progression of human prostate cancer. Int J Cancer.
2013;133(11):2577-2586.

10. Su Q, Xin L. Notch signaling in prostate cancer: refining a therapeutic opportunity. Histol Histopathol. 2016;31(2):149-157.

11. Zayzafoon M, Abdulkadir SA, McDonald JM. Notch signaling and ERK activation are important for the osteomimetic properties of prostate cancer bone metastatic cell lines. J Biol Chem. 2004;279(5):3662-3670.

12. Patrawala L, Calhoun T, Schneider-Broussard R, Zhou J, Claypool K, Tang DG. Side population is enriched in tumorigenic, stem-like cancer cells, whereas ABCG2+ and ABCG2- cancer cells are similarly tumorigenic. Cancer Res. 2005;65(14):6207-6219.

13. Domingo-Domenech J, et al. Suppression of acquired docetaxel resistance in prostate cancer through depletion of Notch- and Hedgehogdependent tumor-initiating cells. Cancer Cell. 2012;22(3):373-388.

14. Wang Z, et al. Down-regulation of Notch-1 is associated with Akt and FoxM1 in inducing cell growth inhibition and apoptosis in prostate cancer cells. J Cell Biochem. 2011;112(1):78-88.

15. Wang Z, et al. Down-regulation of Notch-1 and Jagged-1 inhibits prostate cancer cell growth, migration and invasion, and induces apoptosis via inactivation of Akt, mTOR, and NF- $\mathrm{KB}$ signaling pathways. J Cell Biochem. 2010;109(4):726-736.
16. Yong T, Sun A, Henry MD, Meyers S, Davis JN. Down regulation of CSL activity inhibits cell proliferation in prostate and breast cancer cells. JCell Biochem. 2011;112(9):2340-2351.

17. Kwon OJ, et al. Increased Notch signalling inhibits anoikis and stimulates proliferation of prostate luminal epithelial cells. Nat Commun. 2014;5:4416.

18. Shou J, Ross S, Koeppen H, de Sauvage FJ, Gao WQ. Dynamics of notch expression during murine prostate development and tumorigenesis. Cancer Res. 2001;61(19):7291-7297.

19. Danza $G$, et al. Notch signaling modulates hypoxia-induced neuroendocrine differentiation of human prostate cancer cells. Mol Cancer Res. 2012;10(2):230-238.

20. Lavery DN, Villaronga MA, Walker MM, Patel A, Belandia B, Bevan CL. Repression of androgen receptor activity by HEYL, a third member of the Hairy/Enhancer-of-split-related family of Notch effectors. J Biol Chem. 2011;286(20):17796-17808.

21. Belandia B, Powell SM, Garcia-Pedrero JM, Walker MM, Bevan CL, Parker MG. Hey1, a mediator of notch signaling, is an androgen receptor corepressor. Mol Cell Biol. 2005;25(4):1425-1436.

22. Taylor BS, et al. Integrative genomic profiling of human prostate cancer. Cancer Cell. 2010;18(1):11-22.

23. Sboner A, et al. Molecular sampling of prostate 
cancer: a dilemma for predicting disease progression. BMC Med Genomics. 2010;3:8.

24. Cairns P, et al. Frequent inactivation of PTEN/ MMAC1 in primary prostate cancer. Cancer Res. 1997;57(22):4997-5000.

25. Palomero T, et al. Mutational loss of PTEN induces resistance to NOTCH1 inhibition in T-cell leukemia. Nat Med.2007;13(10):1203-1210.

26. Wang S, et al. Prostate-specific deletion of the murine Pten tumor suppressor gene leads to metastatic prostate cancer. Cancer Cell. 2003;4(3):209-221.

27. Han $\mathrm{H}$, et al. Inducible gene knockout of transcription factor recombination signal binding protein-J reveals its essential role in T versus B lineage decision. Int Immunol. 2002;14(6):637-645.

28. Valdez JM, et al. Notch and TGFbeta form a reciprocal positive regulatory loop that suppresses murine prostate basal stem/progenitor cell activity. Cell Stem Cell. 2012;11(5):676-688.

29. Stanger BZ, Datar R, Murtaugh LC, Melton DA. Direct regulation of intestinal fate by Notch. Proc Natl Acad Sci U S A. 2005;102(35):12443-12448.

30. Jin C, McKeehan K, Wang F. Transgenic mouse with high Cre recombinase activity in all prostate lobes, seminal vesicle, and ductus deferens. Prostate. 2003;57(2):160-164.

31. Muzumdar MD, Tasic B, Miyamichi K, Li L, Luo L. A global double-fluorescent Cre reporter mouse. Genesis. 2007;45(9):593-605.

32. Thompson M, et al. Identification of candidate prostate cancer genes through comparative expression-profiling of seminal vesicle. Prostate. 2008;68(11):1248-1256.

33. Hollier BG, et al. FOXC2 expression links epithelial-mesenchymal transition and stem cell properties in breast cancer. Cancer Res. 2013;73(6):1981-1992.

34. Mulholland DJ, et al. Pten loss and RAS/MAPK activation cooperate to promote EMT and metastasis initiated from prostate cancer stem/progenitor cells. Cancer Res. 2012;72(7):1878-1889.

35. Mani SA, et al. Mesenchyme Forkhead 1 (FOXC2) plays a key role in metastasis and is associated with aggressive basal-like breast cancers. Proc Natl Acad Sci U S A. 2007;104(24):10069-10074.

36. Jang IH, et al. Notch1 acts via Foxc2 to promote definitive hematopoiesis via effects on hemogenic endothelium. Blood. 2015;125(9):1418-1426.
37. Castel D, Mourikis P, Bartels SJ, Brinkman AB, Tajbakhsh S, Stunnenberg HG. Dynamic binding of RBPJ is determined by Notch signaling status. Genes Dev. 2013;27(9):1059-1071.

38. Yu YH, et al. MiR-520h-mediated FOXC2 regulation is critical for inhibition of lung cancer progression by resveratrol. Oncogene. 2013;32(4):431-443.

39. Demehri S, Turkoz A, Kopan R. Epidermal Notch1 loss promotes skin tumorigenesis by impacting the stromal microenvironment. Cancer Cell. 2009;16(1):55-66.

40. Klinakis A, et al. A novel tumour-suppressor function for the Notch pathway in myeloid leukaemia. Nature. 2011;473(7346):230-233.

41. Rampias T, et al. A new tumor suppressor role for the Notch pathway in bladder cancer. Nat Med. 2014;20(10):1199-1205.

42. Zhang Y, Wang Z, Ahmed F, Banerjee S, Li Y, Sarkar FH. Down-regulation of Jagged-1 induces cell growth inhibition and $S$ phase arrest in prostate cancer cells. Int JCancer. 2006;119(9):2071-2077.

43. Mazzone M, et al. Dose-dependent induction of distinct phenotypic responses to Notch pathway activation in mammary epithelial cells. Proc Natl Acad Sci U S A. 2010;107(11):5012-5017.

44. Bailis W, Pear WS. Notch and PI3K: how is the road traveled? Blood. 2012;120(7):1349-1350.

45. Harrison $\mathrm{H}$, et al. Regulation of breast cancer stem cell activity by signaling through the Notch 4 receptor. Cancer Res. 2010;70(2):709-718.

46. Cui H, Kong Y, Xu M, Zhang H. Notch3 functions as a tumor suppressor by controlling cellular senescence. Cancer Res. 2013;73(11):3451-3459.

47. del Alamo D, Rouault H, Schweisguth F. Mechanism and significance of cis-inhibition in Notch signalling. Curr Biol. 2011;21(1):R40-R47.

48. Chen Z, et al. Crucial role of p53-dependent cellular senescence in suppression of Pten-deficient tumorigenesis. Nature. 2005;436(7051):725-730.

49. Dail M, et al. Loss of oncogenic Notch1 with resistance to a PI3K inhibitor in T-cell leukaemia. Nature. 2014;513(7519):512-516.

50. Bin Hafeez B, et al. Targeted knockdown of Notch1 inhibits invasion of human prostate cancer cells concomitant with inhibition of matrix metalloproteinase-9 and urokinase plasminogen activator. Clin Cancer Res. 2009;15(2):452-459.

51. Cui YM, et al. FOXC2 promotes colorectal cancer proliferation through inhibition of FOXO3a and activation of MAPK and AKT signaling pathways. Cancer Lett. 2014;353(1):87-94.

52. Wang J, et al. B-Raf activation cooperates with PTEN loss to drive c-Myc expression in advanced prostate cancer. Cancer Res. 2012;72(18):4765-4776.

53. Cui D, Dai J, Keller JM, Mizokami A, Xia S, Keller ET. Notch pathway inhibition using PF-03084014, a $\gamma$-secretase inhibitor (GSI), enhances the anti-tumor effect of docetaxel in prostate cancer. Clin Cancer Res. 2015;21(20):4619-4629.

54. Fischer KR, et al. Epithelial-to-mesenchymal transition is not required for lung metastasis but contributes to chemoresistance. Nature. 2015;527(7579):472-476.

55. Zheng X, et al. Epithelial-to-mesenchymal transition is dispensable for metastasis but induces chemoresistance in pancreatic cancer. Nature. 2015;527(7579):525-530.

56. Karthaus WR, et al. Identification of multipotent luminal progenitor cells in human prostate organoid cultures. Cell. 2014;159(1):163-175.

57. Gao D, et al. Organoid cultures derived from patients with advanced prostate cancer. Cell. 2014;159(1):176-187.

58. Laczko I, Hudson DL, Freeman A, Feneley MR, Masters JR. Comparison of the zones of the human prostate with the seminal vesicle: morphology, immunohistochemistry, and cell kinetics. Prostate. 2005;62(3):260-266.

59. Siegel R, Ma J, Zou Z, Jemal A. Cancer statistics, 2014. CA Cancer J Clin. 2014;64(1):9-29.

60. Thiel R, Effert P. Primary adenocarcinoma of the seminal vesicles. JUrol. 2002;168(5):1891-1896.

61. Staack A, Donjacour AA, Brody J, Cunha GR, Carroll P. Mouse urogenital development: a practical approach. Differentiation. 2003;71(7):402-413.

62. Choi N, Zhang B, Zhang L, Ittmann M, Xin L. Adult murine prostate basal and luminal cells are self-sustained lineages that can both serve as targets for prostate cancer initiation. Cancer Cell. 2012;21(2):253-265.

63. Xin L, Teitell MA, Lawson DA, Kwon A, Mellinghoff IK, Witte ON. Progression of prostate cancer by synergy of AKT with genotropic and nongenotropic actions of the androgen receptor. Proc Natl Acad Sci U S A. 2006;103(20):7789-7794. 